\title{
Tunable Omnidirectional Band Gap Properties of 1D Plasma Annular Periodic Multilayer Structure Based on an Improved Fibonacci Topological Structure
}

\section{Hong-Mei Peng}

Nanjing university of posts and Telecommunications

\section{Bao-Fei Wan}

Nanjing University of Posts and Telecommunications

\section{Peng-Xiang Wang}

Nanjing University of Posts and Telecommunications

Dan Zhang

Nanjing Forestry University

Hai-Feng Zhang ( $\nabla$ hanlor@163.com )

Nanjing University of Aeronautics and Astronautics

\section{Research Article}

Keywords: plasma cylindrical photonic crystals, Fibonacci, quasi-periodic structure, omnidirectional band gap, transfer matrix method

Posted Date: February 10th, 2021

DOI: https://doi.org/10.21203/rs.3.rs-181473/v1

License: (c) (i) This work is licensed under a Creative Commons Attribution 4.0 International License.

Read Full License 


\title{
Tunable omnidirectional band gap properties of 1D plasma annular periodic multilayer
}

\section{structure based on an improved Fibonacci topological structure}

\author{
Hong-Mei Peng ${ }^{1}$, Bao-Fei Wan ${ }^{1}$, Peng-Xiang Wang ${ }^{1}$, Dan Zhang $^{2}$, and Hai-Feng Zhang ${ }^{1-3 *}$ \\ ${ }^{1}$ College of Electronic and Optical Engineering \& College of Microelectronics, Nanjing University of Posts and Telecommunications, Nanjing \\ 210023, China \\ ${ }^{2}$ College of Information Science and Technology, Nanjing Forestry University, Nanjing 210037, China \\ ${ }^{3}$ State Key Laboratory of Millimeter Waves of Southeast University, Nanjing 210096, China
}

\begin{abstract}
In this paper, the characteristics of the omnidirectional band gap (OBG) for one-dimensional (1D) plasma cylindrical photonic crystals (PCPCs) are based on an improved Fibonacci topological (IFT) structure are studied. The influences of the azimuthal mode number, incident angle, plasma thickness, and plasma frequency on the OBG are discussed. It is concluded that increasing the azimuth modulus can significantly expand the bandwidth of the OBG, and the OBG can be moved to the low-frequency direction by increasing the plasma frequency. In addition, an interesting phenomenon can be found that when the number of azimuthal modes is equal to 2, the TM wave can produce an extra high reflection zone. It provides a theoretical support for designing the narrowband filters without introducing any physical defect layers in the structure.
\end{abstract}

Keyword: plasma cylindrical photonic crystals, Fibonacci, quasi-periodic structure, omnidirectional band gap, transfer matrix method.

\section{Introduction}

Photonic crystals (PCs) [1-3] are a kind of synthetic material, which is formed by the periodic distribution of different mediums in space. When the electromagnetic wave passes through the periodic medium, the Bragg scattering will occur [4, 5], so the PCs can produce the photonic band gaps (PBGs), which is similar to the electronic band gap in the semiconductor. An electromagnetic wave with a specific frequency can be reflected which is located in the PBGs. Thanks to the interesting properties of PBG, PCs can be used to make the reflectors [6] or band-pass filters [7]. However, the features of PBGs [8, 9] for the conventional PCs are affected by their topological structures. As a result, Their PBGs are immutable. For the sake of overcoming such a defect, on the one hand, a large number of special materials have to be introduced, on the other hand, a reasonable structure and parameter optimization can be designed.

At any incident angle, PCs can generate the PBGs for the arbitrary polarization mode, which is called the OBG [10-13]. OBG has a very wide application prospect, which can be used to design many devices, such as waveguides [14], omnidirectional filters [15], and antenna substrates [16]. For those devices, a wider OBG, better performance can be obtained. It has been proved that the bandwidth of the OBG can be extended by changing or further breaking the periodicity of the topology of PCs. In PCs design, the quasi-periodic structure (QPS) [17-20] has attracted more attention. This is because the QPS not only retains the basic physical properties of the period but also its structure is suitable for generating more complex PBGs, providing the possibility of realizing an ultra-wideband OBG compared with the periodic structure. Therefore, the PCs with the QPS formed alternately by dielectric and plasma can improve the performance of PBGs [21, 22]. In 2016, when Srivastava et al were studying the optical properties of high-temperature superconducting cylindrical photonic crystals (HTSCPCs), they found a superpolariton gap for a higher-order azimuthal number that exists in the case of

*Corresponding author at: College of Electronic and Optical Engineering \& College of Microelectronics,Nanjing University of Posts and Telecommunications, Nanjing, 210023 ,China

E-mail: hanlor@163.com ( Hai-Feng Zhang) 
the TM wave [23]. In 2009, Chen et al studied the wave characteristics of single-negative materials cylindrical photonic crystals (CPCs) and found that the TE wave produced additional high reflection bands and that the PBG had a great dependence on the initial radius [24]. In 2013, Hu et al derived the reflection and transmission formulas of the CPCs under H-polarization [25]. In 2013, Aly et al investigated that the number of PBGs can be controlled by changing the thickness of the dielectric layer when they were studying the HTSCPCs [26]. However, no one has done researches on the OBG characteristics of CPCs. Therefore, in this paper, a 1D PCPC with IFT structure is presented, whose attributes of OBGs are studied. In the modern lasers system of vertical emission [27], the CPCs produces the complete band gaps, improves the constraint of transverse propagation [28], and have potential application in the optical communication and optoelectronics [23]. Consequently, research on the CPCs is essential.

In the presented paper, a 1D PCPC based on the IFT structure is studied, which is composed of the plasma and dielectric alternately distributed in space. Compared with the metal-dielectric PCs, the wave traits of plasma PCs can be tuned. Since the wave characteristics of plasma depend on the plasma frequency, incident wave frequency and collision frequency, the wave features of plasma can be adjusted by changing these parameters. In the process of studying the process reflection spectra, we found that the reflection properties of the PCPCs based on an IFT depend on the azimuthal mode number and other parameters. In addition, for the TM wave, an additional high emission band can be found when the azimuthal mode number is 2 . This extra high reflection band can be used to make a narrow band, which provides a feasible method to design a narrow band transmission filter without introducing any defect layer to break the periodicity of structure [24, 29]. We figure out that a larger azimuthal mode number can obtain a wider OBG. This provides an effective scheme for the realization of the ultra-wideband OBGs and the ultra-wideband PBGs. In this paper, the influences of different azimuthal modulus on the reflection characteristics of PCPCs based on an IFT is studied, afterwards, the effects of the incidence angle, plasma thickness, and plasma frequency on the reflective property are discussed.

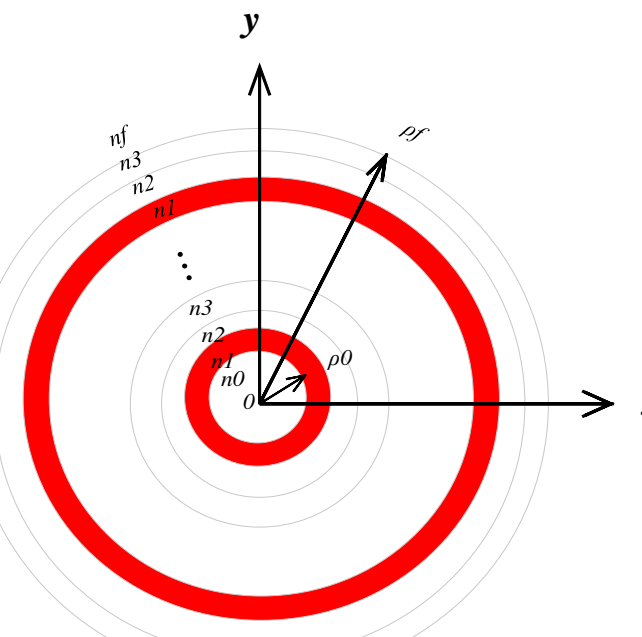

Fig.1 The cross-section view of a 1D PCPCs based on the IFT structure .

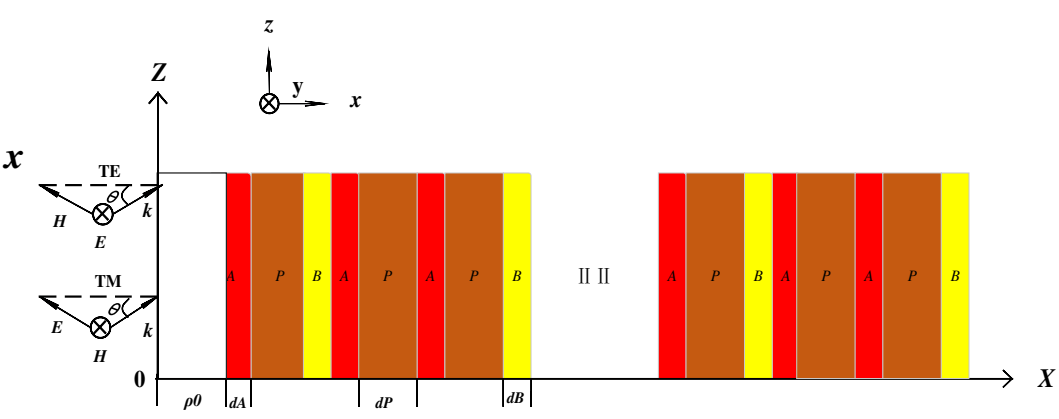

Fig.2 A cross-section view of the medium distribution of the 1D PCPCs periodic multilayer structure based on an IFT viewed from the axis. A and $\mathrm{B}$ are common media layers, and $\mathrm{P}$ is plasma layer. Electromagnetic waves are incident from free space.

\section{Simulation model and calculation method}

The Fibonacci sequence 0-level sequence can be expressed as ( $\mathrm{BBAB} . . . .$.$) , the first-level sequence can be written as$ ( $\mathrm{ABAABA} . . . . .$.$) , the second-level sequence can be marked as (ABAABABAAB......). This paper uses the Fibonacci second-order$

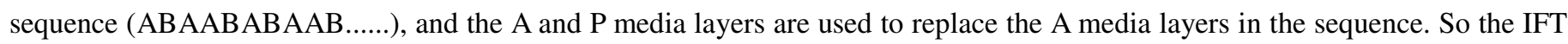
structure is (APBAPAPBAPBAPAPB......), for the sake of increasing the selective selection the period is 5. The model of the 1D PCPCs with IFT structure is shown in Fig.1. Fig.2 gives a cross-sectional view of the medium distribution of the 1D PCPCs 
periodic multilayer structure with IFT viewed from the axis. The electromagnetic waves are incident from the free space. The model with air as background, so $n_{f}=n_{0}=1$, and let the starting radius of $\rho_{0}=\mathrm{d}, \mathrm{d}$ is a normalization constant. A and B represent ordinary dielectric layers, and P represents a plasma layer. Their thicknesses are described by $d_{A}, d_{B}, d_{P}$, where $d_{A}=d_{B}=0.08 \mathrm{~d}$, and $d_{p}=0.2 \mathrm{~d}$. In the following calculations, the frequency region is normalized by $2 \pi c / d$, and $c$ is the speed of light in a vacuum. The dielectric constant is $\varepsilon_{i}(i=\mathrm{A}, \mathrm{B}$ or $\mathrm{P})$, where $\varepsilon_{A}=4, \varepsilon_{B}=1$, and $\varepsilon_{p}$ of the plasma can be expressed as [30]:

$$
\varepsilon_{p}=1-\frac{\omega_{p}^{2}}{\omega^{2}+j v_{c} \omega}
$$

where $\omega$ represents the angular frequency of the incident electromagnetic wave, $v_{c}$ represents the collision frequency of the plasma and $\omega_{p}$ represents plasma frequency. The initial parameters are set as $v_{c}=\pi \times 10^{-5} \times \mathrm{c} / \mathrm{d} \mathrm{H}, \omega_{p}=2 \pi \times 0.2 \times \mathrm{c} / \mathrm{d} \mathrm{rad} / \mathrm{s}$.

Respectively, suppose the electromagnetic field is related to time $\mathrm{e}^{(j \omega t)}$, and two Maxwell equations for the passive region are given below

$$
\begin{aligned}
& \nabla \times E=-j \omega \mu H . \\
& \nabla \times H=j \omega \varepsilon E
\end{aligned}
$$

There are two kinds of possible waves in cylindrical coordinates: TE and TM waves. the $E_{z}, H_{\phi}$ and $H_{\rho}$ are non-zero and can be represented by the following three equations for the TE wave.

$$
\begin{gathered}
\frac{1}{\rho} \frac{\partial E_{z}}{\partial \phi}=-j \omega \mu H_{\rho}, \\
\frac{\partial E_{Z}}{\partial \rho}=j \omega \mu H_{\phi}, \\
\frac{\partial\left(\rho H_{\phi}\right)}{\partial \rho}-\frac{\partial H_{\rho}}{\partial \phi}=j \omega \varepsilon \rho E_{z} .
\end{gathered}
$$

From Eq.(4), the equation of $E_{Z}$ can be obtained by removing $H_{\phi}$ and $H_{\rho}$

$$
\rho \frac{\partial}{\partial \rho}\left(\rho \frac{\partial E_{z}}{\partial \rho}\right)-\rho^{2} \frac{1}{\mu} \frac{\partial \mu}{\partial \rho} \frac{\partial E_{z}}{\partial \rho}+\frac{\partial}{\partial \phi}\left(\frac{\partial E_{z}}{\partial \phi}\right)+\omega^{2} \mu \varepsilon \rho^{2} E_{z}=0 \text {. }
$$

With Eq.(5), the following equation can be acquired by separating the variables

$$
E_{z}(\rho, \phi)=V(\rho) \varphi(\phi)=\left[A J_{m}(k \rho)+B Y_{m}(k \rho)\right] e^{j m \phi}
$$

where $\mathrm{A}$ and $\mathrm{B}$ are two constants, $Y_{m}$ is a Numann function, $J_{m}$ is a Bessel function, $m$ is the azimuthal number, and $k=\omega(\varepsilon \mu)^{1 / 2} \cos (\theta)$ is the wave number of the medium, respectively. With Eq. (4), the azimuth part of the magnetic field can be expressed as

$$
H_{\phi}(\rho, \varphi)=U(\rho) \phi(\varphi)=-j p\left[A j_{m}^{\prime}(k \rho)+B Y_{m}^{\prime}(k \rho)\right] e^{j m \phi}
$$

where $p=(\varepsilon / \mu)^{1 / 2} \cos (\theta)$ is the intrinsic admittance of the medium. In the light of Eqs.(5) and (6), we can deduce the single-layer matrix relationship related to electric and magnetic fields. For example, as for the first matrix, we can establish a single-layer matrix relation related to electric and magnetic fields. The first matrix $M I$ (with refractive index $n_{l}$ and interfaces at $\rho=\rho_{0}$ and $\rho_{l}$ ) can be written as

$$
\left[\frac{V(\rho 1)}{U(\rho 1)}\right]=M 1\left[\frac{V(\rho 0)}{U(\rho 0)}\right]
$$


The first layer matrix $M 1$ can be expressed as

$$
M 1=\left[\begin{array}{ll}
m 11 & m 12 \\
m 21 & m 22
\end{array}\right]
$$

The elements in $M 1$ can be specifically expressed as [23, 29]

$$
\begin{aligned}
& m 11=\frac{\pi}{2} k_{1} \rho_{0}\left[Y_{\mathrm{m}}^{\prime}\left(k_{1} \rho_{0}\right) J_{m}\left(k_{1} \rho_{1}\right)-J_{m}^{\prime}\left(k_{1} \rho_{0}\right) Y_{m}\left(k_{1} \rho_{1}\right)\right], \\
& m 12=j \frac{\pi}{2} \frac{k_{1}}{p_{1}} \rho_{0}\left[J_{m}\left(k_{1} \rho_{0}\right) Y_{\mathrm{m}}\left(k_{1} \rho_{1}\right)-Y_{m}\left(k_{1} \rho_{0}\right) J_{m}\left(k_{1} \rho_{1}\right)\right], \\
& m 21=-j \frac{\pi}{2} k_{1} p_{1} \rho_{0}\left[Y_{m}^{\prime}\left(k_{1} \rho_{0}\right) J_{m}^{\prime}\left(k_{1} \rho_{1}\right)-J_{m}^{\prime}\left(k_{1} \rho_{0}\right) Y_{m}^{\prime}\left(k_{1} \rho_{1}\right)\right], \\
& m 22=\frac{\pi}{2} k_{1} \rho_{0}\left[J_{m}\left(k_{1} \rho_{0}\right) Y_{m}^{\prime}\left(k_{1} \rho_{1}\right)-Y_{m}\left(k_{1} \rho_{0}\right) J_{m}^{\prime}\left(k_{1} \rho_{1}\right)\right] .
\end{aligned}
$$

where $p=\left(\varepsilon_{l} / \mu_{1}\right)^{1 / 2} \cos \left(\theta_{l}\right)$. It can be seen from the formula that the size of matrix elements is related to the radius of the two interfaces. For layer $i$, its transfer matrix $M i$ can be replaced by

$$
\rho_{0} \rightarrow \rho_{\mathrm{i}-1}, \rho_{1} \rightarrow \rho_{i}, k_{1} \rightarrow k_{i}=\omega \sqrt{\mu_{i} \varepsilon_{i}} \cos \left(\theta_{i}\right), p_{1} \rightarrow p_{i}=\sqrt{\varepsilon_{i} / \mu_{i}} \cos \left(\theta_{i}\right)
$$

For the 1D PCPCs based on IFT structure with period $N$, we have a total of $8 N$ matrices. Forasmuch, the total matrix $M$ can be expressed by

$$
\begin{gathered}
{\left[\begin{array}{l}
V\left(\rho_{f}\right) \\
U\left(\rho_{f}\right)
\end{array}\right]=M\left[\begin{array}{l}
V\left(\rho_{0}\right) \\
U\left(\rho_{0}\right)
\end{array}\right] .} \\
M=\left[\begin{array}{ll}
M_{11} & M_{12} \\
M_{21} & M_{22}
\end{array}\right]=M_{8 N} \ldots . . M_{3} M_{2} M_{1}
\end{gathered}
$$

The transmission matrix of the 1D PCPCs with IFT structure is related to the half meridian. Therefore, its must be calculated one by one.

$$
C_{m l}^{1,2}=\frac{H_{m}^{1,2}\left(k_{l} \rho_{l}\right)}{H_{m}^{1,2}\left(k_{l} \rho_{l}\right)} \quad l=0, f
$$

Among them, $H^{l}{ }_{m}$ and $\mathrm{H}^{2}$ are the first Hankel function and the second kind of Hankel function, respectively. For the starting medium and the last medium $p=\left(\varepsilon_{0} / \mu_{0}\right)^{1 / 2} \cos \left(\theta_{0}\right)$ and $p=\left(\varepsilon_{f} / \mu_{f}\right)^{1 / 2} \cos \left(\theta_{f}\right)$. The reflectivity $R$ and transmissivity $T$ are expressed as follows

$$
\begin{aligned}
& R=r d \times r d^{*}=|r d|^{2} . \\
& T=t d \times t d^{*}=|t d|^{2} .
\end{aligned}
$$

Where

$$
r d=\frac{\left(M_{12}^{\prime}+j p_{0} C_{m 0}^{2} M_{11}^{\prime}\right)-j p_{f} C_{m f}^{2}\left(M_{22}^{\prime}+j p_{0} C_{m 0}^{2} M_{12}^{\prime}\right)}{\left(-j p_{0} C_{m 0}^{1} M_{11}^{\prime}-M_{21}^{\prime}\right)-j p_{f} C_{m f}^{2}\left(-j p_{0} C_{m 0}^{1} M_{12}^{\prime}-M_{22}^{\prime}\right)}
$$




$$
t d=\frac{4 \sqrt{\varepsilon_{0} / \mu_{0}}}{\pi K \rho_{0} H_{m}^{2}\left(k_{0} \rho_{0}\right) H_{m}^{1}\left(k_{0} \rho_{0}\right)\left[\left(-j p_{0} C_{m 0}^{1} M_{11}^{\prime}-M_{21}^{\prime}\right)-j p_{f} C_{m f}^{2}\left(-j p_{0} C_{m 0}^{2} M_{12}^{\prime}-M_{22}^{\prime}\right)\right]} .
$$

Where $M_{11}^{\prime}, M_{12}^{\prime}, M_{21}^{\prime}, M_{22}^{\prime}$ are the matrix elements of the inverse matrix of $M, K=\omega\left(\varepsilon_{0} \mu_{0}\right)^{1 / 2}$ is used to represent the free-space wave number. Replace the relevant parameters in the above CPCs formula as follows

$$
\varepsilon \leftrightarrow \mu, j \leftrightarrow-j,
$$

the transmission matrix formula of TM wave can be obtained. Special attention should be paid to TM wave $\quad p=\sqrt{\frac{\mu}{\varepsilon}} \frac{1}{\cos (\theta)}$

\section{Results and discussion}
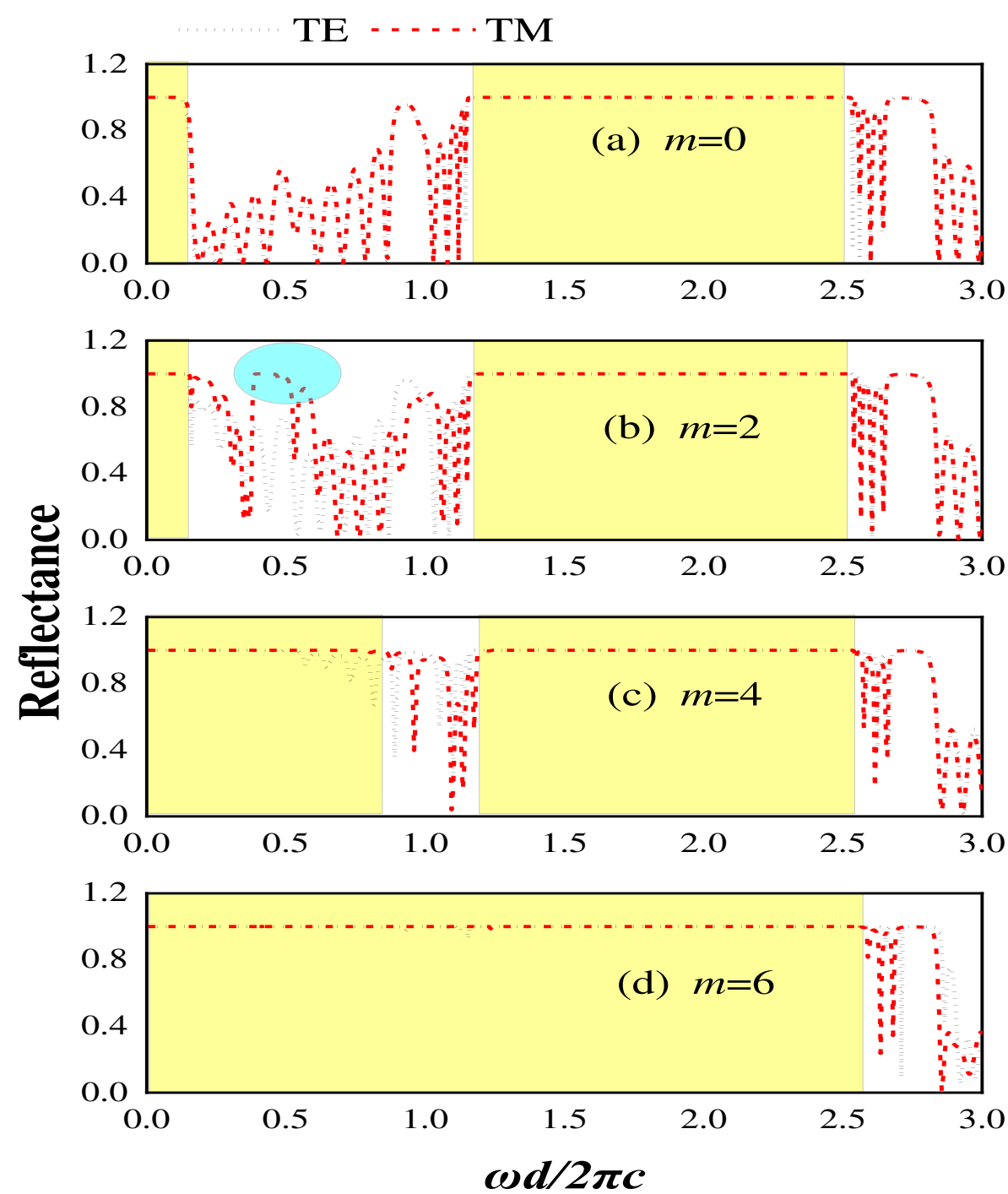

Fig.3 The diagrams of the reflectivities of the TM and TE waves for 1D PCPCs based on the IFT structure with different $m$ when $\theta=60^{\circ}$. (a) $m=0$, (b) $m=2$, (c) $m=4$, and (d) $m=6$.

Fig. 3 shows the reflection spectra of the TE and TM waves of 1D PCPCs based on the IFT structure with different $m$ under the above parameters. In Fig.3(a), the reflectivity of the TE wave will be decreased suddenly only when the frequencies are 1.1493 $(2 \pi c / d)$ and $2.5467(2 \pi c / d)$, but the TE and TM waves almost overlap in other regions. It can be obtained that the two reflection spectra are almost the same, which shows that at $m=0$, the geometric difference of the curved interface of the cylinder has almost the same effect on the optical properties of the TE and TM waves. However, with the increase of $m$, there is a significant 
difference between the TE and TM waves in the non-reflection region. When $m=6$, the TE and TM waves form the PBG in $0 \sim 2.5754(2 \pi c / d)$, and they are overlapped. Surprisingly, when $m=2$, an extra ultra-high reflection band (blue area) appears in the TM wave, and its bandwidth is $0.1505(2 \pi c / d)$. In the case of $m=4$, this extra high reflection band overlaps with the PBG in front and disappears. Fig.3 strongly and forcefully illustrates that the bandwidth of PBG in the high-frequency region hardly changes with the change of $m$. On the contrary, the duration of PBG in the low-frequency region is amplified with the value of $m$ is enlarged and is moved towards a high-frequency direction. As expected in Fig.3, the bandwidth of PBG in the high-frequency region is not affected by the increase of $m$ and its bandwidth is $1.376(2 \pi c / d)$. On the contrary, the bandwidth of PBG in the low-frequency region is enlarged when $m$ is magnified and moved to a higher frequency direction. While $m=6$, it connects with PBG in the high-frequency region to form a larger PBG with the bandwidth of $2.5754(2 \pi c / d)$. So, the PBG will be broadened when $m$ is amplified. To more intuitively see what changes PBG and OBG will have with the transform of $m$, Fig. 4 is plotted.

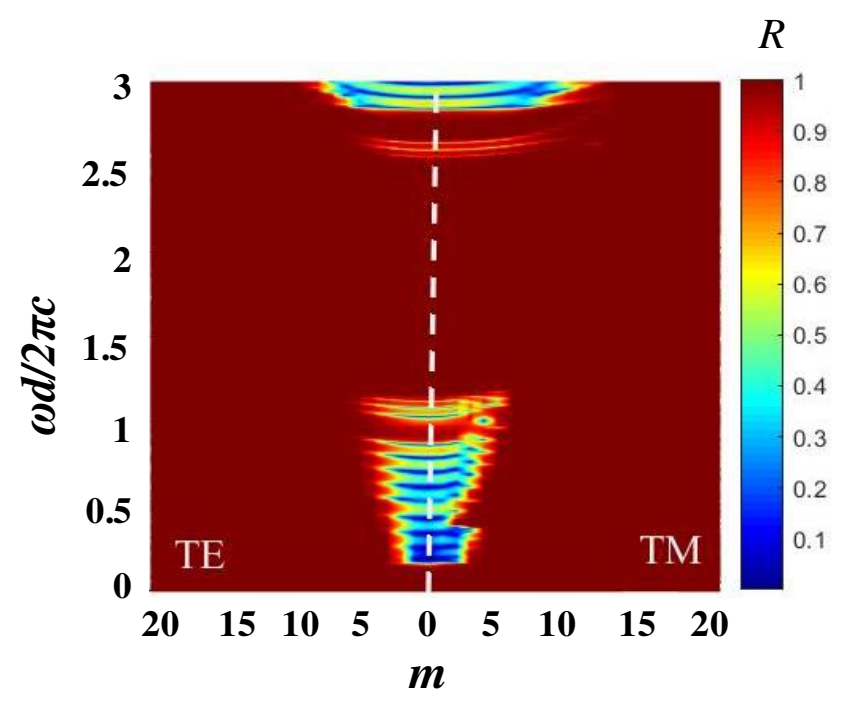

(a)

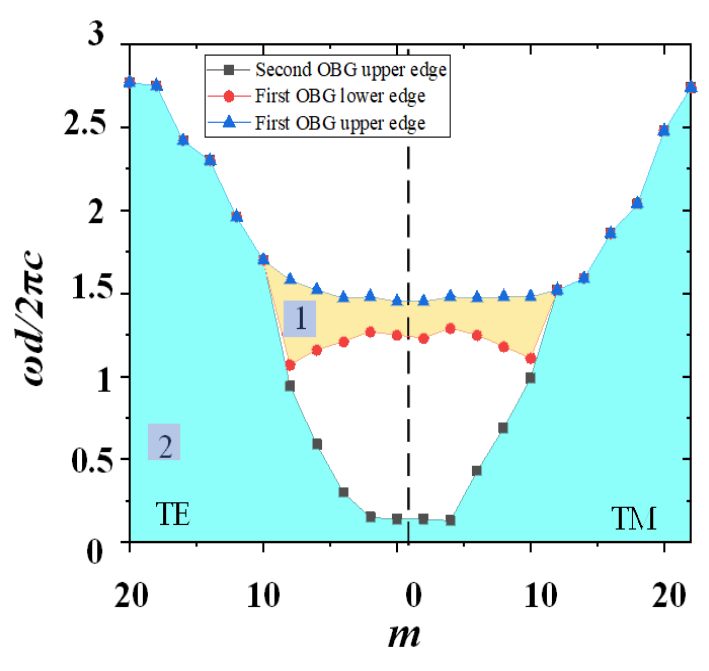

(b)

Fig.4 Testimony of the relationship between $R$ and $m$ when $\theta=60^{\circ}$. (a) three-dimensional (3D) top view of $R$ diagram under different $m$, and (b) diagram of OBG at different $m$.

The relationships between PBG and $m$, OBG, and $m$ are given in Fig.4 when the incident angle $\theta=60^{\circ}$. In Fig.4 (a), when $m$ turns from 0 to 20, the PBG bandwidths of both TE and TM waves are significantly broadened. The PBG bandwidths of the TE and TM waves can be significantly affected by $m$. Fig.3(d) proves that at $m=6$, almost all TE and TM waves form PBGs below $2.5396(2 \pi c / d)$. While $m=13$, the TE and TM waves form a bigger PBG between $0(2 \pi c / d)$ and $3(2 \pi c / d)$. The changing trends of the TE and TM waves are roughly the same, as shown in Fig.4. For the first OBG (brown area), with the value of $m$ enlarged, the upper edge of the TM wave is moved slowly to the high-frequency direction, while the lower edge is moved to the low-frequency direction, the bandwidth of the first OBG is increased. For the second OBG (blue area), with the magnification of $m$, the upper edge is rapidly moved to the high-frequency direction. In the case of $m=10$, the first OBG overlaps with the second OBG to form a comparatively large OBG. At $m=20$, the bandwidth of the bigger OBG is enlarged to $2.77(2 \pi c / d)$. Therefore, a larger $m$ can obtain the larger PBG and OBG, and increasing the value of $m$ is beneficial to expand the bandwidths of OBGs.

Fig.5 Illustration of the reflection curves of TE and TM waves when the incident angle is $0^{\circ}, 30^{\circ}, 60^{\circ}$ and $85^{\circ}$. As plotted in Fig.5(a), if $\theta=0^{\circ}$ (normal incidence), the frequency range of the PBG bandwidth in the high frequency area is $0.9701 \sim 1.4288$ $(2 \pi c / d)$ and the bandwidth is $0.4587(2 \pi c / d)$; The bandwidth of PBG in the low-frequency range is $0.1245(2 \pi c / d)$, its frequency range is $0 \sim 0.1245\left(2 \pi c / d\right.$ ). At $\theta=60^{\circ}$ (see Fig.5(c)), the frequency range of PBG in the high-frequency region is $1.1923 \sim 2.5398$ $(2 \pi c / d)$, and the width is $1.3473(2 \pi c / d)$; The PBG in the low-frequency region is $0 \sim 0.1603(2 \pi c / d)$ and its bandwidth is 0.1603 $(2 \pi c / d)$. Available from the above data, when the incident angle is enlarged from $0^{\circ}$ to $60^{\circ}$, the bandwidth of high-frequency region PBG is amplified $0.8886(2 \pi c / d)$, and the starting point of PBG is moved to high-frequency direction $0.2222(2 \pi c / d)$ and the bandwidth of low-frequency region PBG is magnified $0.0358(2 \pi c / d)$. It can be concluded that the bandwidth of PBG increases with the enlarging of the incident angle and moves towards a high-frequency direction. From Fig.5 we can see that the 
TM wave produces an extra high reflection band (the blue areas) in the low-frequency region. As mentioned in Fig.5(b), $\theta=30^{\circ}$ the starting frequency of this PBG is $0.2248(2 \pi c / d)$, whose width is $0.072(2 \pi c / d)$. In Fig.5(d), At $\theta=85^{\circ}, 0.9558(2 \pi c / d)$ is its starting position and the bandwidth of the PBG is $0.2652(2 \pi c / d)$. Therefore, a larger incident angle can get a larger PBG and its location is moved to the high-frequency region.

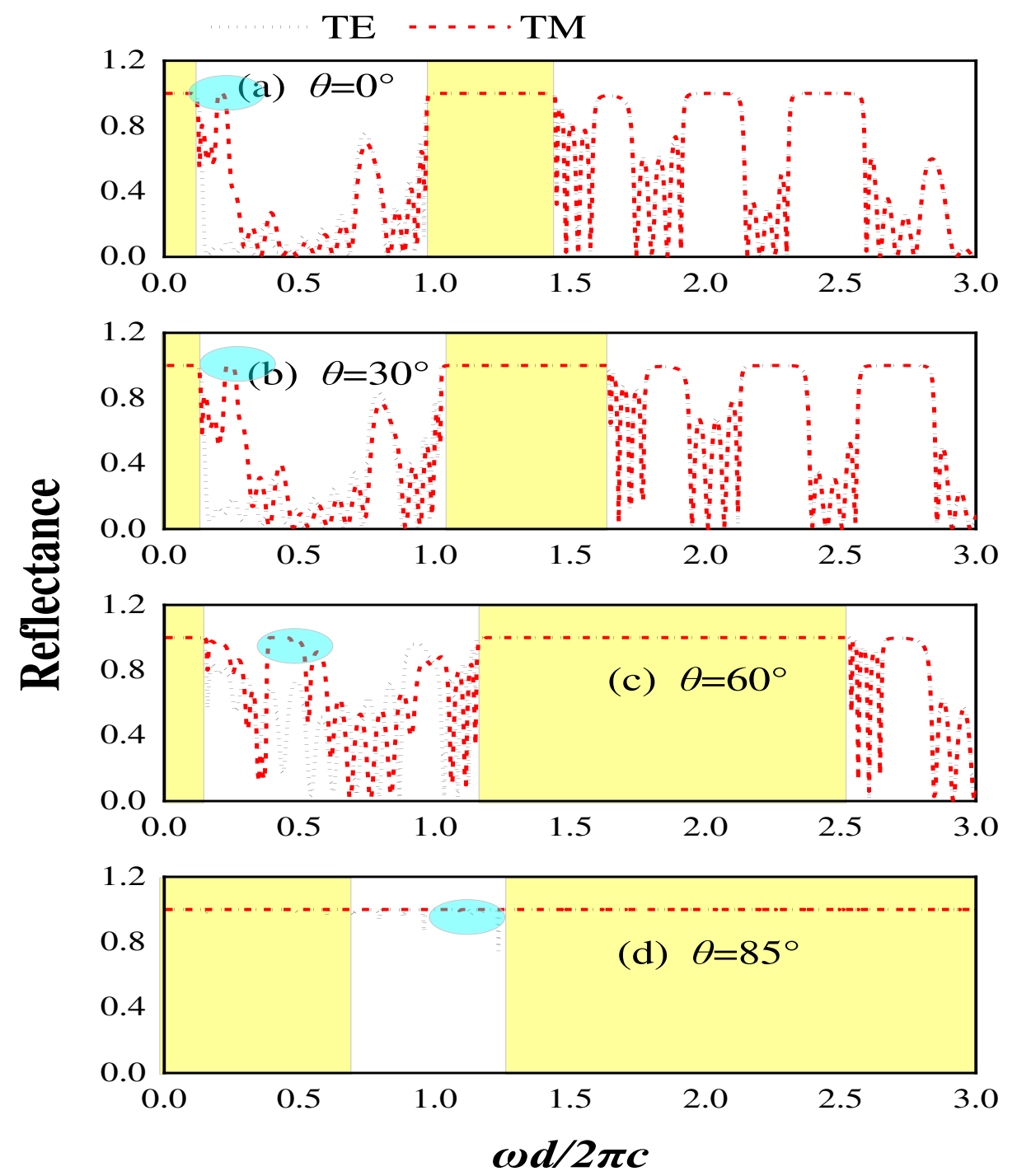

Fig.5 The reflection curves of the TM and TE waves for 1D PCPCs based on the IFT structure with different $\theta$ when $m=2$.
(a) $\theta=0^{\circ}$
(b) $\theta=30^{\circ}$
(c) $\theta=60^{\circ}$, and (d) $\theta=85^{\circ}$.

Fig.6 is used to make known the OBG diagram of 1D PCPCs based on an improved Fibonacci topology, and the region between the white lines represents the OBG. In Fig.6, the OBG frequency ranges of the TE and TM waves are $0 \sim 0.1625$ (2 $2 \pi / d$ ) and $1.1900 \sim 1.4690(2 \pi c / d)$, and their bandwidths are $0.1625(2 \pi c / d)$ and $0.2790(2 \pi c / d)$. Besides, the OBG region of both polarized waves shifts to the higher frequencies when $\theta$ is increased. 


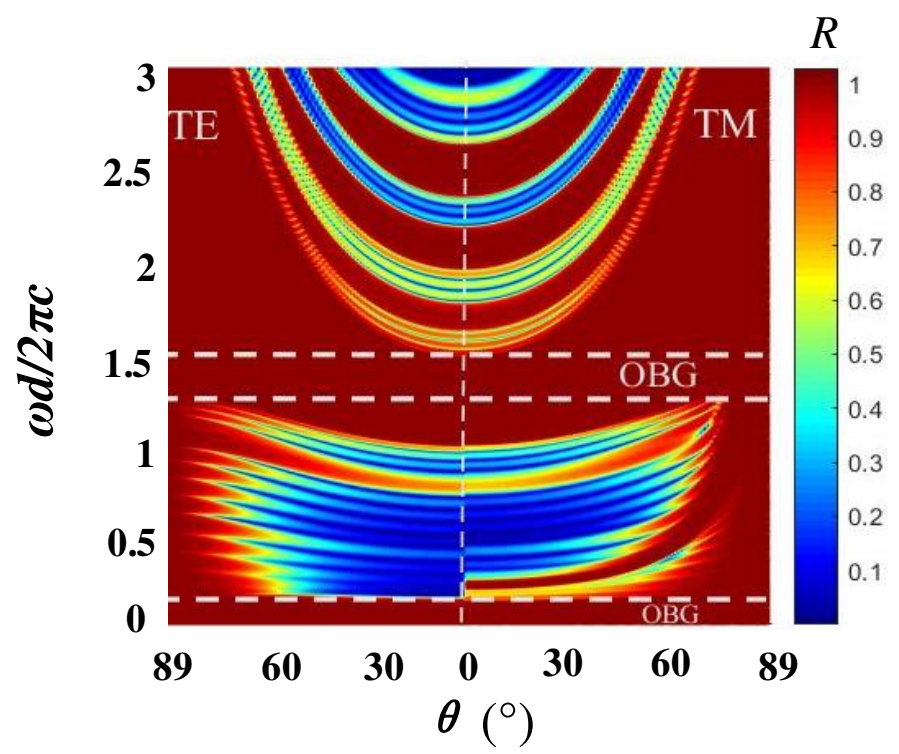

Fig.6 3D top view of $R$ diagram under different $\theta$, illustration of the relationship between $\theta$ and $R$ when $m=2$.

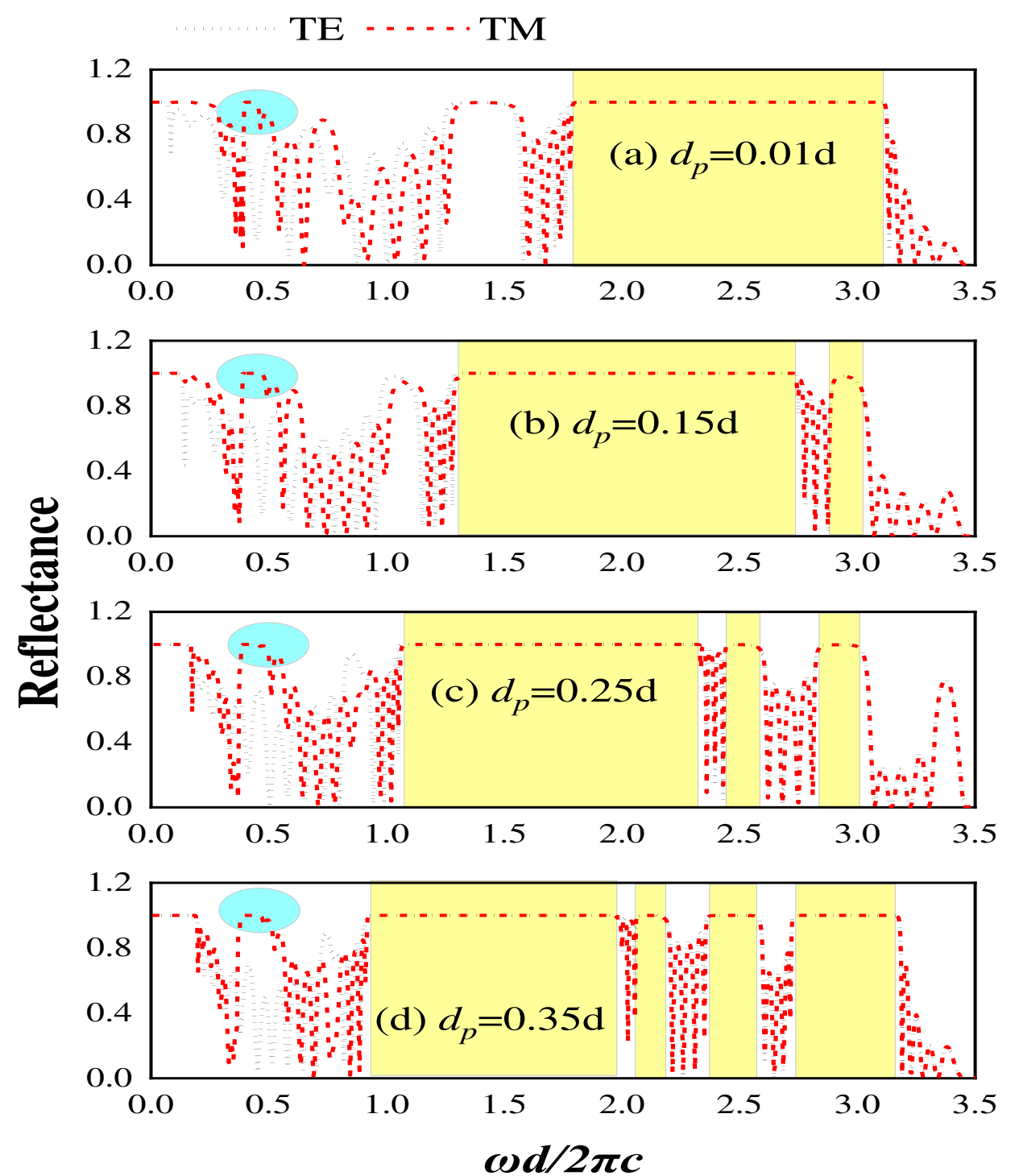

Fig.7 The reflection spectra of the TM and TE waves for 1D PCPCs based on the IFT structure with different $d_{p}$ when $\theta=60^{\circ}$ and $m=2$. (a) $d_{p}=0.01 \mathrm{~d}$, (b) $d_{p}=0.15 \mathrm{~d}$, (c) $d_{p}=0.25 \mathrm{~d}$, and (d) $d_{p}=0.35 \mathrm{~d}$. 
Fig.7 provides the relationship between the reflection spectra of 1D PCPCs based on an improved Fibonacci topology and different plasma thickness $d_{p}$ when the electromagnetic wave is incident at $60^{\circ}$. As $d_{p}$ accumulates from $0.01 \mathrm{~d}$ to $0.35 \mathrm{~d}$, the main PBG frequency range is shifted from $1.7923 \sim 3.1300(2 \pi c / d)$ to $0.9312 \sim 1.9846(2 \pi c / d)$, and the bandwidth is abated from $1.3377(2 \pi c / d)$ to $1.0534(2 \pi c / d)$. Therefore, with the accumulation of $d_{p}$, the frequency of occurrence of the main PBG is reduced and the bandwidth is decreased. As shown in Fig.7, by enlarging $d_{p}$, the new PBGs appear. So, we can control the number of PBGs by changing the thickness of $d_{p}$. The main PBG in Fig.7(b) increases temporarily because the main PBG is fused with the PBG in front of it. In the low frequency region of the TM wave, an additional high reflection band (the blue areas) will be produced, which is confirmed by Fig.7. The width increases from $0.0418(2 \pi c / d)$ to $0.125(2 \pi c / d)$ because of the enlargement of $d_{p}$. Thus, it can be concluded that with the amplify of $d_{p}$, the magnify of the ultrahigh reflection band is very slow.

To clearly observe the OBG phenomena of the TM and TE waves, the reflectivity spectrograms of two polarized waves with different $d_{p}$ are given in Fig.8. Fig.8 proves that the value of $d_{p}$ has a significant effect on the PBGs of the TE and TM waves when $\theta=60^{\circ}$, but has no contribution to the PBG broadening. While $d_{p}$ is increased, the upper and lower edges of the PBGs in the TE and TM waves move to the low-frequency direction. In Fig.8, the PBG bandwidths of the TE and TM waves increase slowly with the $d_{p}$ aggrandized, reaches the maximum near $0.1 \mathrm{~d}$, and then decrease with the increase of $d_{p}$, and at the $d_{p}$ is $0.45 \mathrm{~d}$ the bandwidth is reduced to $0(2 \pi c / d)$. In Fig.8(b), there are two OBGs of the TE and TM waves in the frequency range of $0 \sim 3(2 \pi c / d)$. The first OBG (the blue area) and PBG have the same transform trend. In the case of $d_{p}=0.45 \mathrm{~d}$, the first OBG is diminished to 0 ( $2 \pi c / d$ ). For the second OBG (the brown area), when $d_{p}$ is in the range of $0.01 \mathrm{~d} \sim 0.05 \mathrm{~d}$, the TE wave bandwidth is close to 0.1 ( $2 \pi c / d$ ), whlie the remaining bandwidth is around $0.2(2 \pi c / d)$. The second OBG bandwidth is almost unchanged for TM wave, and it is at around $0.2(2 \pi c / d)$. In summary, increasing the $d_{p}$ has little effect on the expansion of OBG, while magnifying the $d_{p}$ can be moved PBG and OBG to the low-frequency region. This feature can be used to reduce the operating frequency of the device.

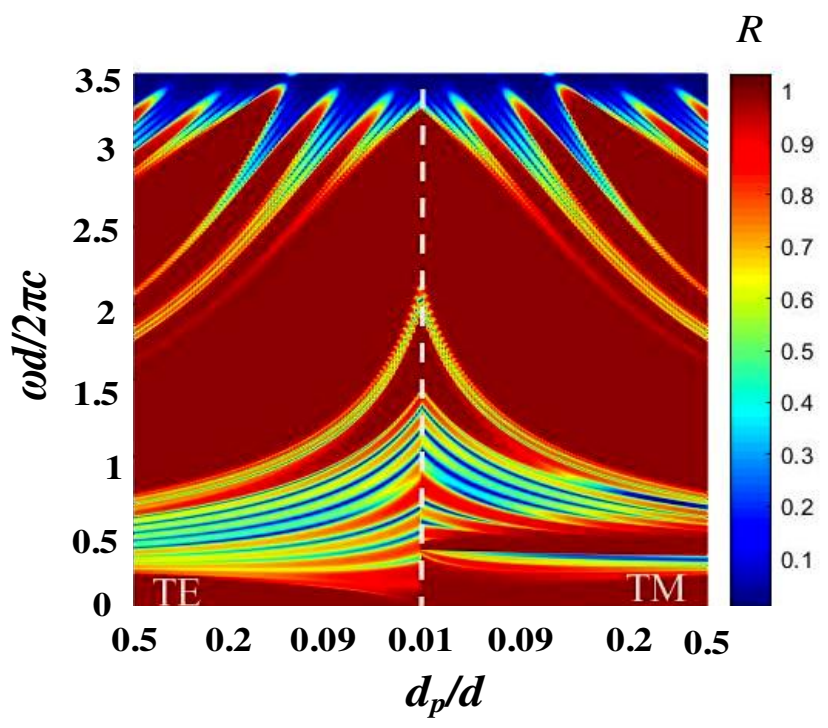

(a)

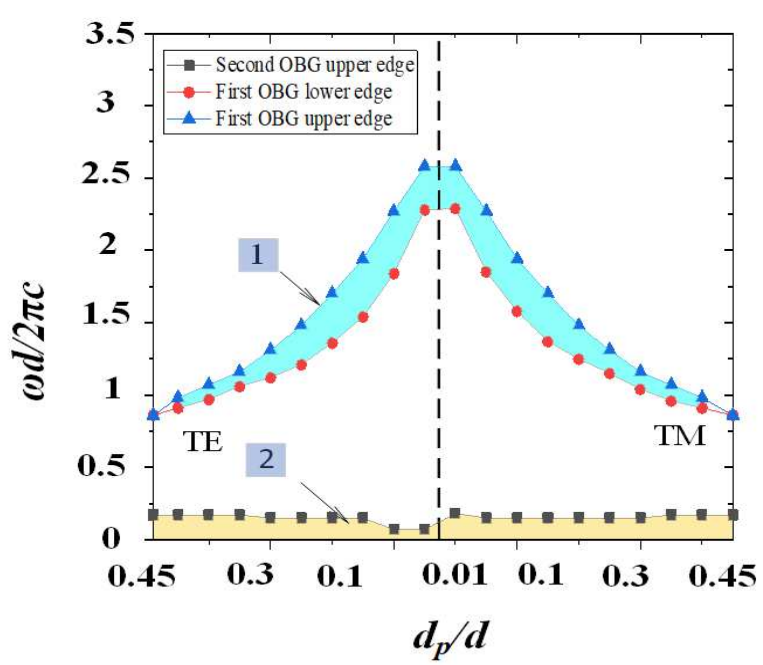

(b)

Fig.8 Diagram of the relationship between $d_{p}$ and $R$ when $\theta=60^{\circ}$ and $m=2$, (a) 3D top view of $R$ diagram under different $d_{p} / d$, and (b) diagram of OBG at different $d_{p} / d$.

When incident angle $\theta=60^{\circ}$, the relationship between reflectivity spectra of different $\omega_{p}$ is revealed in Fig.9. Fig.9 demonstrates that the bandwidth of PBG in the high-frequency region of the TE or TM wave is hardly altered with the $\omega_{p}$ enlarged. At $\omega_{p}=0.01(2 \pi c / d)$, the bandwidth of PBG in the high-frequency region is $1.3616(2 \pi c / d)$, and the width of PBG in the high-frequency region is $1.3902(2 \pi c / d)$ in $\omega_{p}=0.35(2 \pi c / d)$, it has only been changed by $0.0286(2 \pi c / d)$. However, in the low-frequency region, the relationship between PBG and $\omega_{p}$ will have opposite characteristics. The PBG bandwidth is 0.1102 $(2 \pi c / d)$ at $\omega_{p}=0.15(2 \pi c / d)$; When $\omega_{p}=0.35(2 \pi c / d)$, its width is $0.2607(2 \pi c / d)$, and the bandwidth is widened $0.1505(2 \pi c / d)$. In addition, an additional high reflection band (the blue areas) is found in the TM wave. Fig.10(a) certificate this point more strongly. With $\omega_{p}$ altered, the bandwidth of the high reflection band is barely changed. Its starting point will shift to the high-frequency 
direction because of the $\omega_{p}$ increased. Its starting position is $0.2965(2 \pi c / d)$ in the case of $\omega_{p}=0.15(2 \pi c / d)$; While $\omega_{p}=0.35$ $(2 \pi c / d)$, its starting position is $0.6763(2 \pi c / d)$. The $\omega_{p}$ from $0.15(2 \pi c / d)$ to $0.35(2 \pi c / d)$, the starting position of PBG is shifted by $0.3798(2 \pi c / d)$. For the sake of better understanding, the relationships between PBG and $\omega_{p}$, OBG, and $\omega_{p}$ Fig.10 is given. As expected in Fig.10(a), the change of $\omega_{p}$ will not affect the PBG in the high-frequency region, on the contrary, the PBG in the low-frequency region is increased with the increase of $\omega_{p}$. The conclusion that the change of $\omega_{p}$ does not affect the bandwidth of the first OBG (the blue area), but the second OBG (the brown area) is enlarged in the form of $\omega_{p}$ amplification is proved by Fig. 10 . To sum up, it can be concluded that it is effective for widening OBG as the $\omega_{p}$ is increased, while the frequency range should be controlled well, that only works in the region below $0.5(2 \pi c / d)$.

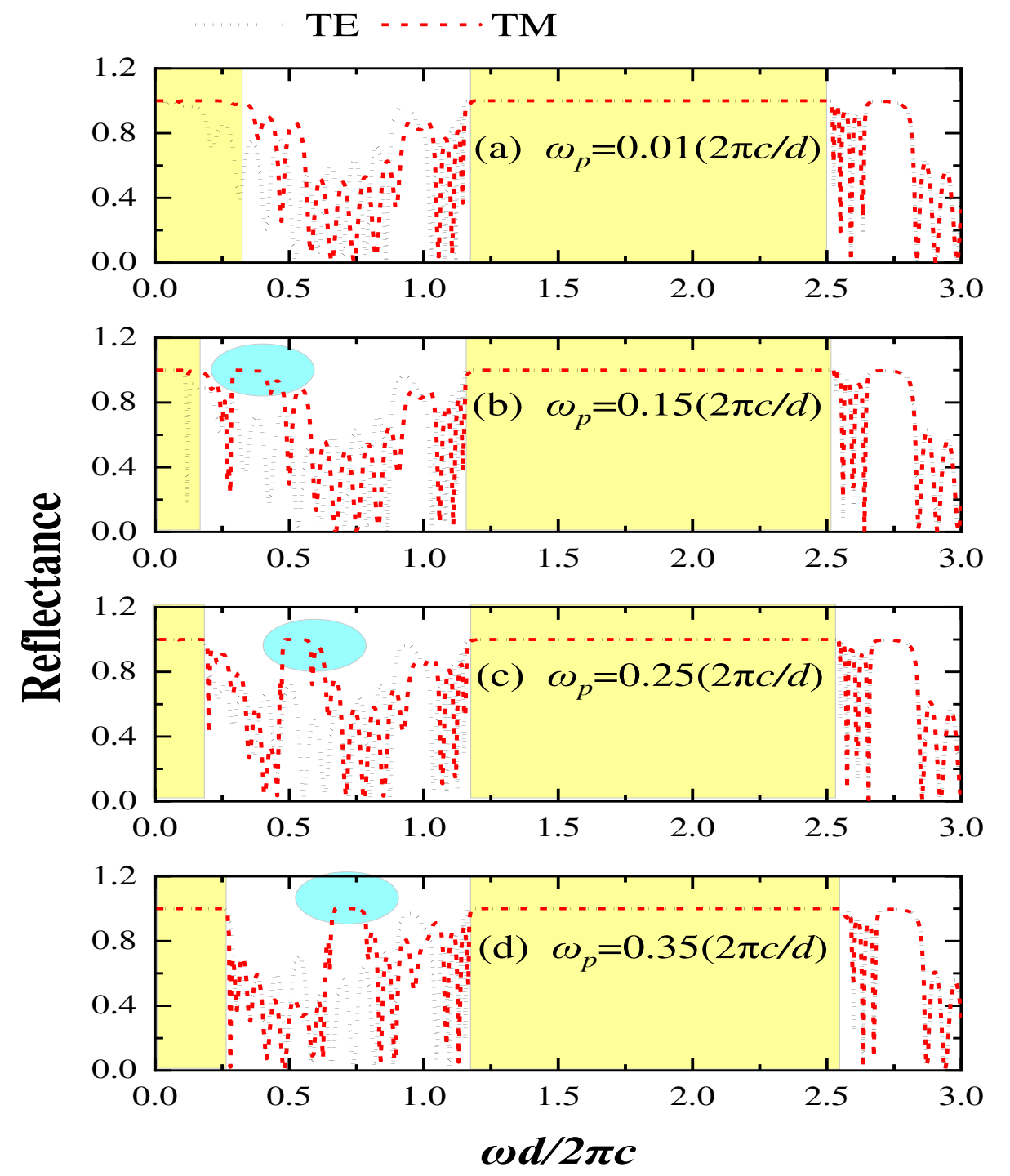

Fig.9 The reflection curves of the TM and TE waves for 1D PCPCs based on IFT structure with different $\omega_{p}$ when $\theta=60^{\circ}$ and $m=2$. (a) $\omega_{p}=0.01(2 \pi c / d)$, (b) $\omega_{p}=0.15(2 \pi c / d)$, (c) $\omega_{p}=0.25(2 \pi c / d)$, and (d) $\omega_{p}=0.35(2 \pi c / d)$. 


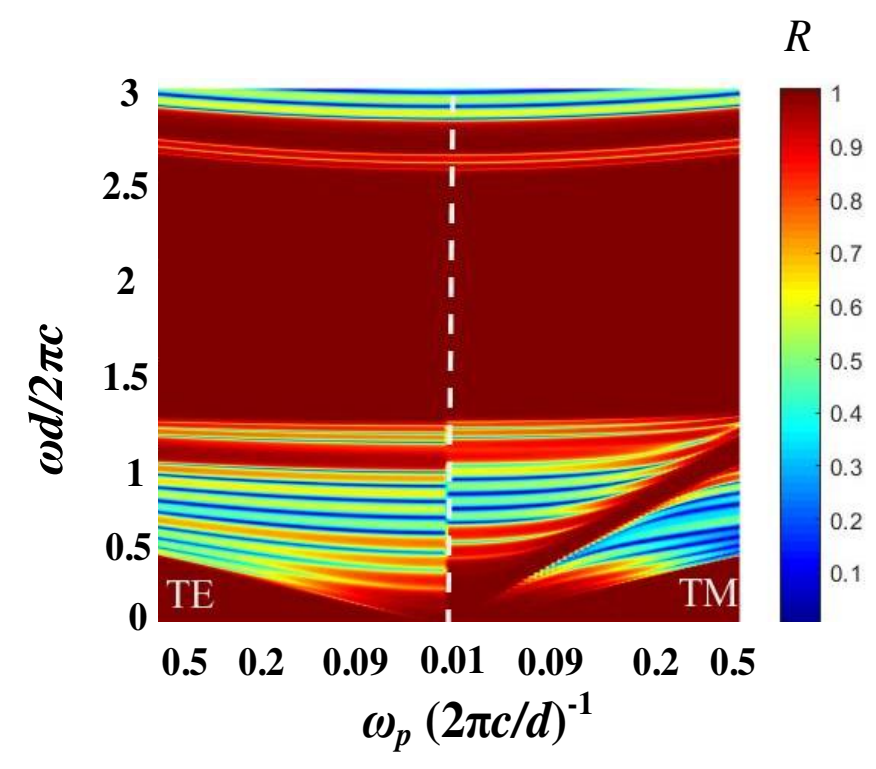

(a)

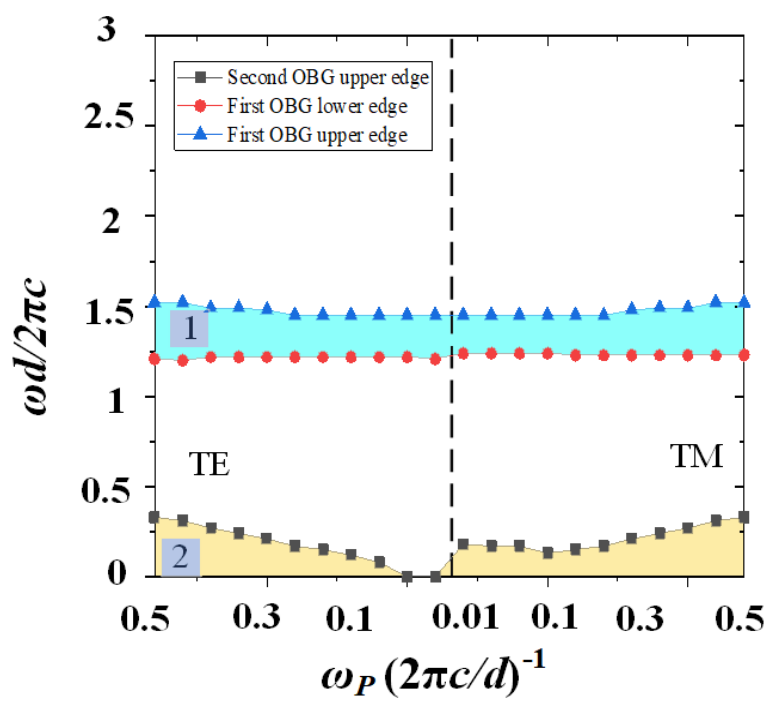

(b)

Fig.10 Confirm the truth of the relationship between $\omega_{p}$ and $R$ when $\theta=60^{\circ}$ and $m=2$. (a) 3D top view of $R$ diagram under different $\omega_{p},(\mathrm{~b})$ the diagram of OBG at different $\omega_{p}$

\section{Conclusion}

In this paper, we propose a model of 1D PCPCs with IFT structure and study their optical properties. Because the parting structure breaks the spatial symmetry of the conventional PCs, it brings some interesting phenomena. In this paper, the effects of $m, \theta, d_{p}$, and $\omega_{p}$ on the optical properties of 1D PCPCs are discussed. We can conclude that it is beneficial to expand the bandwidth of OBG with the value of $m$ increased. Besides, the OBG regions for both polarized waves shift to higher frequencies when $\theta$ is amplified. Therefore, the ultra-wideband OBG and the ultra-wideband PBG can be realized by magnifying $m$ and incident angle $\theta$ properly. With the enlargement of $\omega_{p}$, the position of OBG will move to the low-frequency direction, so the $\omega_{p}$ can be increased to realize the low-frequency omnidirectional reflectors, but $\omega_{p}$ cannot be infinitely amplified, and it is better not to exceed $0.3(2 \pi c / d)$. In addition, there is an unexpected discovery, when $m=2$, the TM wave will produce an extra high reflection band, which magnifies with the increase of $\theta$ and $d_{p}$. The special features make it possible for the 1D PCPCs based on the improved Fibonacci topology to be used for designing a narrowband transmission filter without inserting any physical defect layer in the structure. These interesting results will contribute to the design of the multifunctional devices integrated with OBG in theory and practical applications.

\section{Acknowledgments}

This work was supported by the Open Research Program in China's State Key Laboratory of Millimeter Waves (Grant No. K201927).

\section{References}

[1] . Rajesh V, Nair R, Vijaya. "Photonic crystal sensors: An overview". Progress in Quantum Electronics. 34 89-134 (2010)

[2] . Giampaolo Pitruzzello and Thomas F. Krauss. "Photonic crystal resonances for sensing and imaging". Journal of Optics. 105259.R1(2018)

[3] . Cho Tung Yip, Haitao Huang, Limin Zhou, Keyu Xie et al. "Direct and Seamless Coupling of TiO 2 Nanotube Photonic Crystal to Dye-Sensitized Solar Cell: A Single-Step Approach". Advanced Materials. 23(47) 5624-5628(2011)

[4] . J. J. Wierer, M. R. Krames, J. E. Epler, N. F. Gardner, M. G. Craford et al. " InGaN/GaN quantum-well heterostructure light-emitting diodes employing photonic crystal structures". Applied Physics Letters. 843885 (2004)

[5] . Hua Xiao, Duanzheng Yao. "Optical limitation in two-dimensional nonlinear photonic crystal with triangular lattice". Physics 
Letters A. 359 723-727(2006)

[6] . Alexandros A, Liles,Kapil Debnath et al. "Lithographic wavelength control of an external cavity laser with a silicon photonic crystal cavity-based resonant reflector". Optics Letters Letter. 41(5) 253382(2016)

[7] . Hai-Xia Qiang, Li-Yong Jiang, Xiang-Yin Li, Wei Jia. "Different kinds of band-pass filters based on one-dimensional photonic crystal heterostructures". Optik. 122 1836-1839 (2011)

[8] . Huan Liu, Jianquan Yao, Degang Xu, and Peng Wang. "Characteristics of photonic band gaps in woodpile three-dimensional terahertz photonic crystals". Microwave Theory and Tech. 50 910-928 (2002).

[9] . V.A. Tolmachev, T.S. Perova1, R.A. Moore1. "Method of construction of composite one dimensional photonic crystal with extended photonic band gaps". Optics Express. 13(21) 8433-8441(2005)

[10] . Wulbern, Jan Hendrik, Schmidt, Markus, Eich, Manfred et al. "Omnidirectional photonic band gap in polymer photonic crystal slabs". Applied Physics Letters. 91(22) 221104-221104-3(2007)

[11] . Vipin Kumar, Khundrakpam Saratchandra Singh et al."Broadening of Omnidirectional Photonic Band Gap in Si Based One Dimensional Photonic Crystals".Progress In Electromagnetics Research M. 14 101-111(2011)

[12] . Z. Wang, D. Liu. "A few points on omnidirectional band gaps in one-dimensional photonic crystals". Applied Physics B. 86(3) 473-476(2007)

[13] . Yunkun Zhao, Yuping Zhang, Xiaohan Guo, Maodong Liu et al. "Tunable omnidirectional photonic band gap of one-dimensional photonic crystals containing Dirac semimetals". Journal of Applied Physics. 122223108 (2017)

[14] . Masaya Notomi, Akihiko Shinya, Eiichi Kuramochi, Satoshi Mitsug et al. " Understanding Post-Adoptive Agile Usage an Exploratory Cross-Case analysis". IEICE Transactions on Electronics E. 87C(3) 398-408(2004)

[15] . Young-Ki Ha, Y.-C. Yang, J.-E. Kim, H. Y. Park, C.-S. Kee, H. Lim, J.-C. Lee. "Tunable omnidirectional reflflection bands and defect modes of a one-dimensional photonic band gap structure with liquid crystals". Applied Physics Letters 79(1) 15-17(2001)

[16] . D Lusk, I Abdulhalim, F Placido. "Omnidirectional reflection from Fibonacci quasi-periodic one-dimensional photonic crystal". Optics Communications. 198 273-279(2001)

[17] . Yu Ma, Hai-Feng Zhang, Cai-Xing Hu. "Tunable omnidirectional band gap and polarization splitting in one-dimensional magnetized plasma photonic crystals with a quasi-periodic topological structure". Journal of Optics. 22 025101(2020)

[18] . Sreejith, K. PMathew, Vincent. "Investigation of Transmission Properties in One-Dimensional Quasi-periodic Superconducting Photonic Crystal". Journal of Superconductivity and Novel Magnetism. 31(6) 1-6(2017)

[19] . Xuyang Xiao, Runping Chen. "Study of Omnidirectional Reection Bandgap Extension in One-Dimensional Quasi-Periodic Metallic Photonic Crystal". 10( 6 ) 1550088(2015)

[20] . Yuping Zhang, Zhixin Wu, Yanyan Cao, Huiyun Zhang. "Optical properties of one-dimensional Fibonacci quasi-periodic graphene photonic crystal". Optics Communications. 338(2015)

[21] . Zhang, Hai-Feng, Zhen, Jian-Pin, He, Wen-Ping. "Omnidirectional photonic band gaps enhanced by Fibonacci quasiperiodic one-dimensional ternary plasma photonic crystals". Optik. 124(20) 4182-4187(2013)

[22] . Hai-Feng Zhang, Shao-Bin Liu, Xiang-Kun Kong et al. "Enhancement of omnidirectional photonic band gaps in one-dimensional dielectric plasma photonic crystals with a matching layer". Physics of Plasmas. 19(2) 022103 (2012)

[23] . Sanjeev K. Srivastava, Alireza Aghajamali. "Investigation of Reflectance Properties in 1D Ternary Annular Photonic Crystal Containing Semiconductor and High-Tc Superconductor". Journal of Superconductivity and Novel Magnetism. 29 1423-1431(2016)

[24] . Mei-Soong Chen, Chien-Jang Wu, Tzong-Jer Yang. "Wave properties of an annular periodic multilayer structure containing the single-negative materials". Physics Letters A. 3733594 - 3600(2009)

[25] . Chung An Hu, Chien Jang Wu, zong Jer Yang, Su Lin Yang."Analysis of optical properties in cylindrical dielectric photonic crystal". Optics Communications 291424 - 434(2013)

[26] . Arafa H. Aly, Walied Sabra, Hussein A. Elsayed. "Dielectric and Superconducting Photonic Crystals". J Supercond Nov Magn 26 553-560(2013)

[27] . Mei-Soong Chen, Chien-Jang Wu, Tzong-Jer Yang. " Narrowband reflection-and-transmission filter in an annular defective 
photonic crystal containing an ultrathin metallic film". Optics Communications. 2853143 - 3149(2012)

[28] . Schmidt-Grund Rüdiger, Gühne Tobia, Hochmuth Holger et al. "Cylindric resonators with coaxial Brag reflectors". Proceedings of SPIE - The International Society for Optical Engineering. 6038 603827(2006)

[29] . Mei-Soong Chena, Chien-Jang Wu, Tzong-Jer Yang. "Optical properties of a superconducting annular periodic multilayer structure". Solid State Communications. 149 (2009) 1888 - 1893

[30] . Chun-zao Li, Shao-bin Liu, Xiang-kun Kong, Hai-feng Zhang et al. "A Novel Comb-Like Plasma Photonic Crystal Filter in the Presence of Evanescent Wave". IEEE Transactions on Plasma Science. 39(10) 1969-1973 (2011) 
Figures

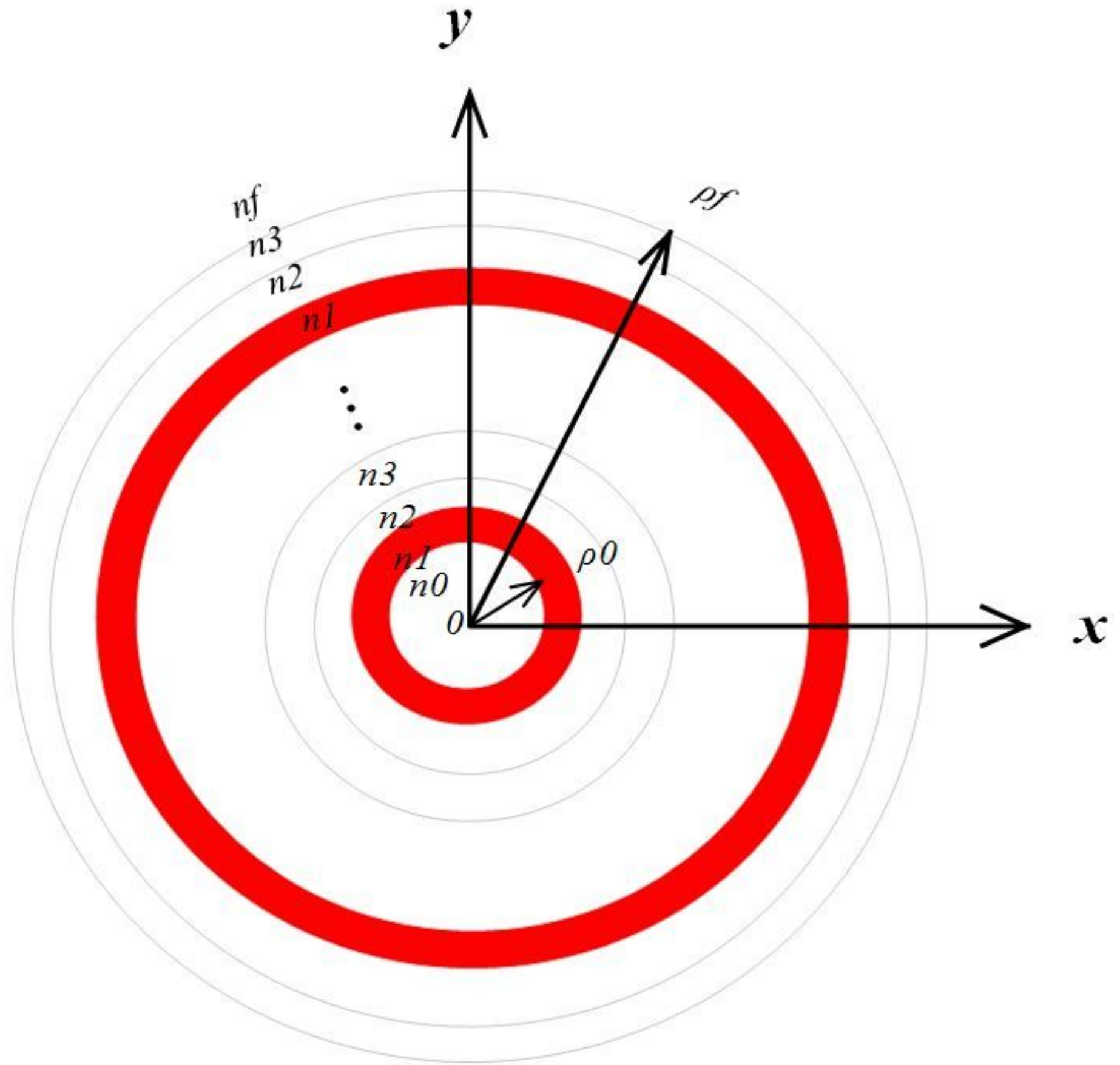

Figure 1

The cross-section view of a 1D PCPCs based on the IFT structure. 


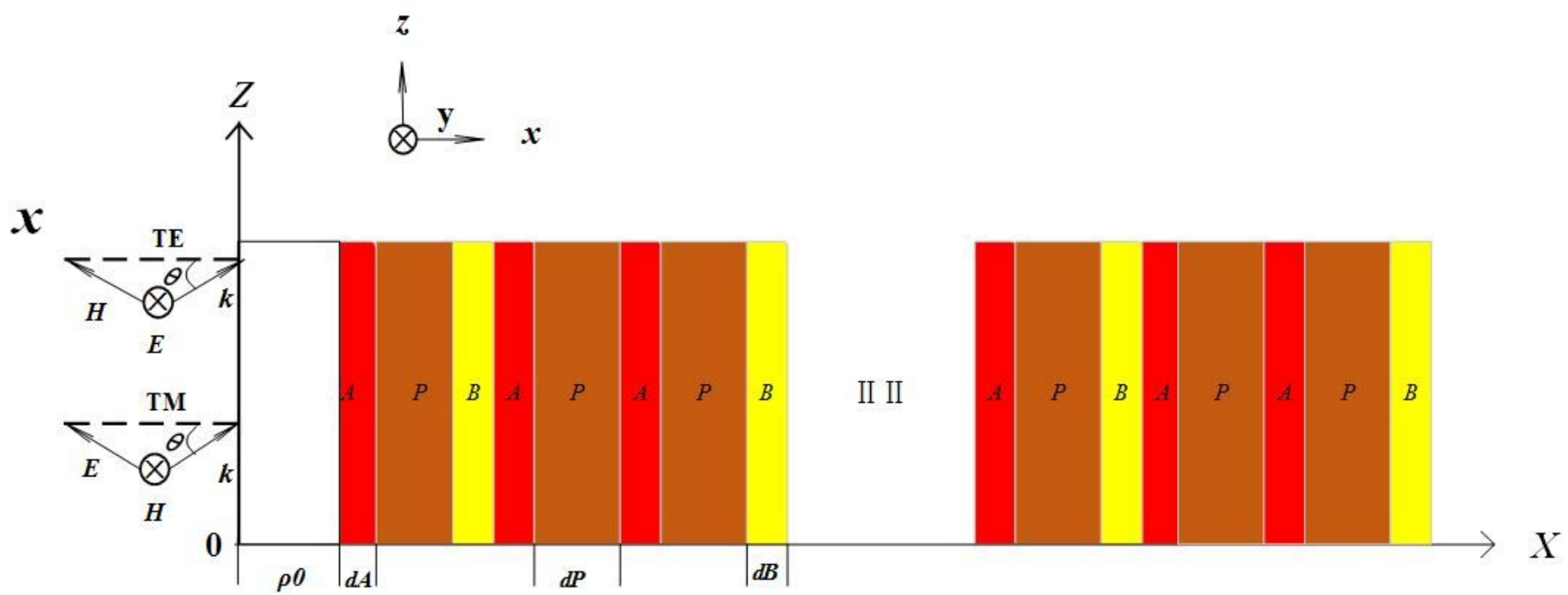

Figure 2

A cross-section view of the medium distribution of the 1D PCPCs periodic multilayer structure based on an IFT viewed from the axis. A and B are common media layers, and P is plasma layer. Electromagnetic waves are incident from free space. 

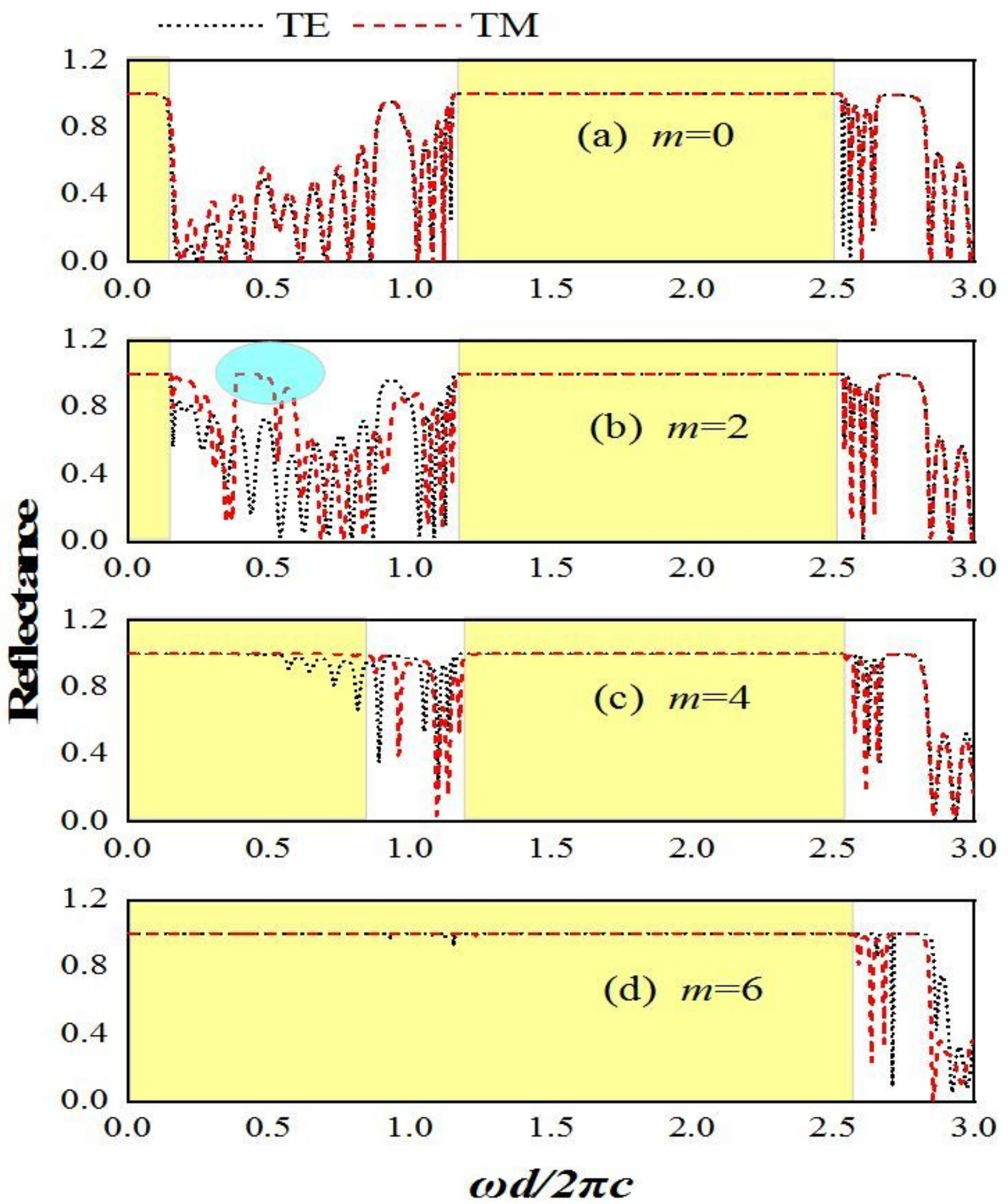

Figure 3

The diagrams of the reflectivities of the TM and TE waves for 1D PCPCs based on the IFT structure with different $m$ when $\theta=60^{\circ}$. (a) $m=0$, (b) $m=2$, (c) $m=4$, and (d) $m=6$. 


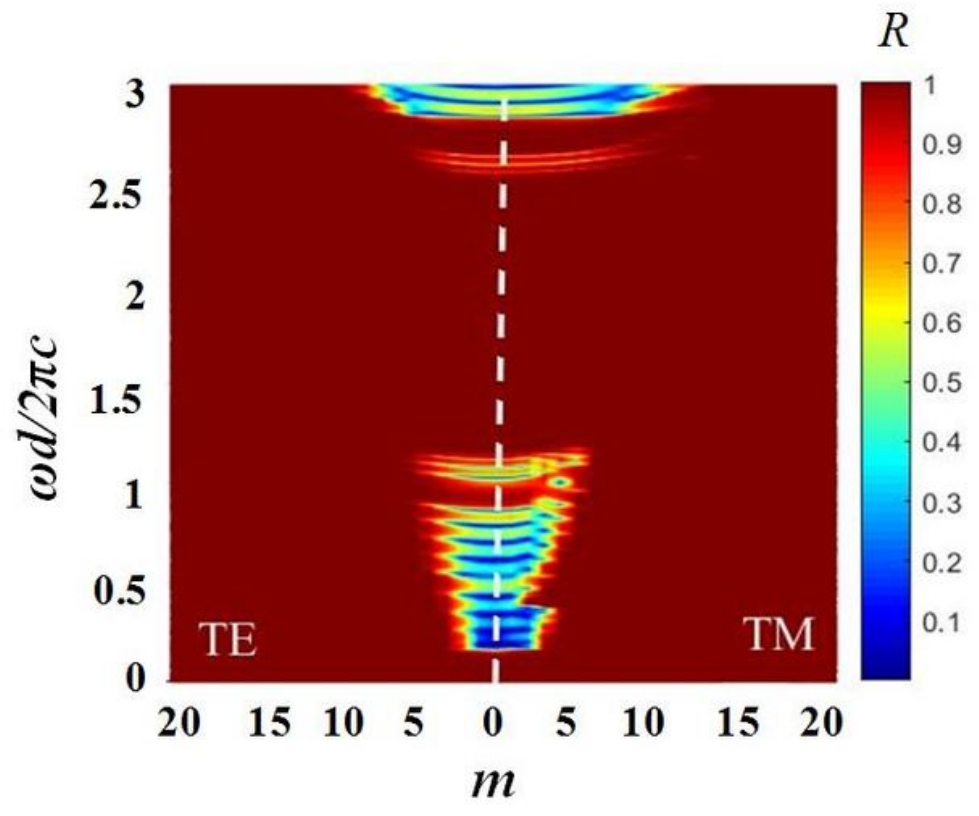

(a)

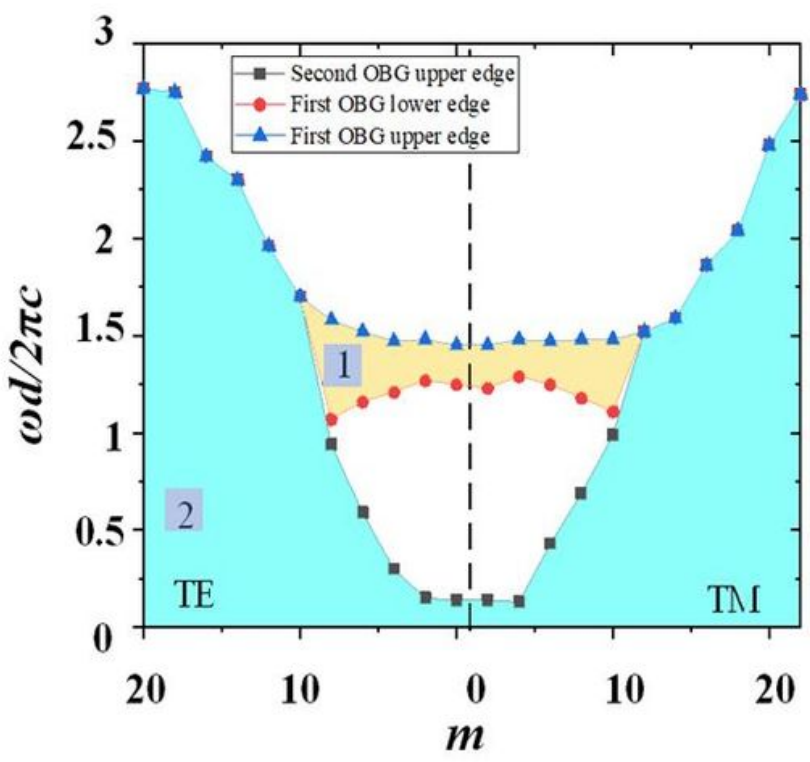

(b)

\section{Figure 4}

Testimony of the relationship between $R$ and $m$ when $\theta=60^{\circ}$. (a) three-dimensional (3D) top view of $R$ diagram under different $\mathrm{m}$, and (b) diagram of $\mathrm{OBG}$ at different $\mathrm{m}$. 

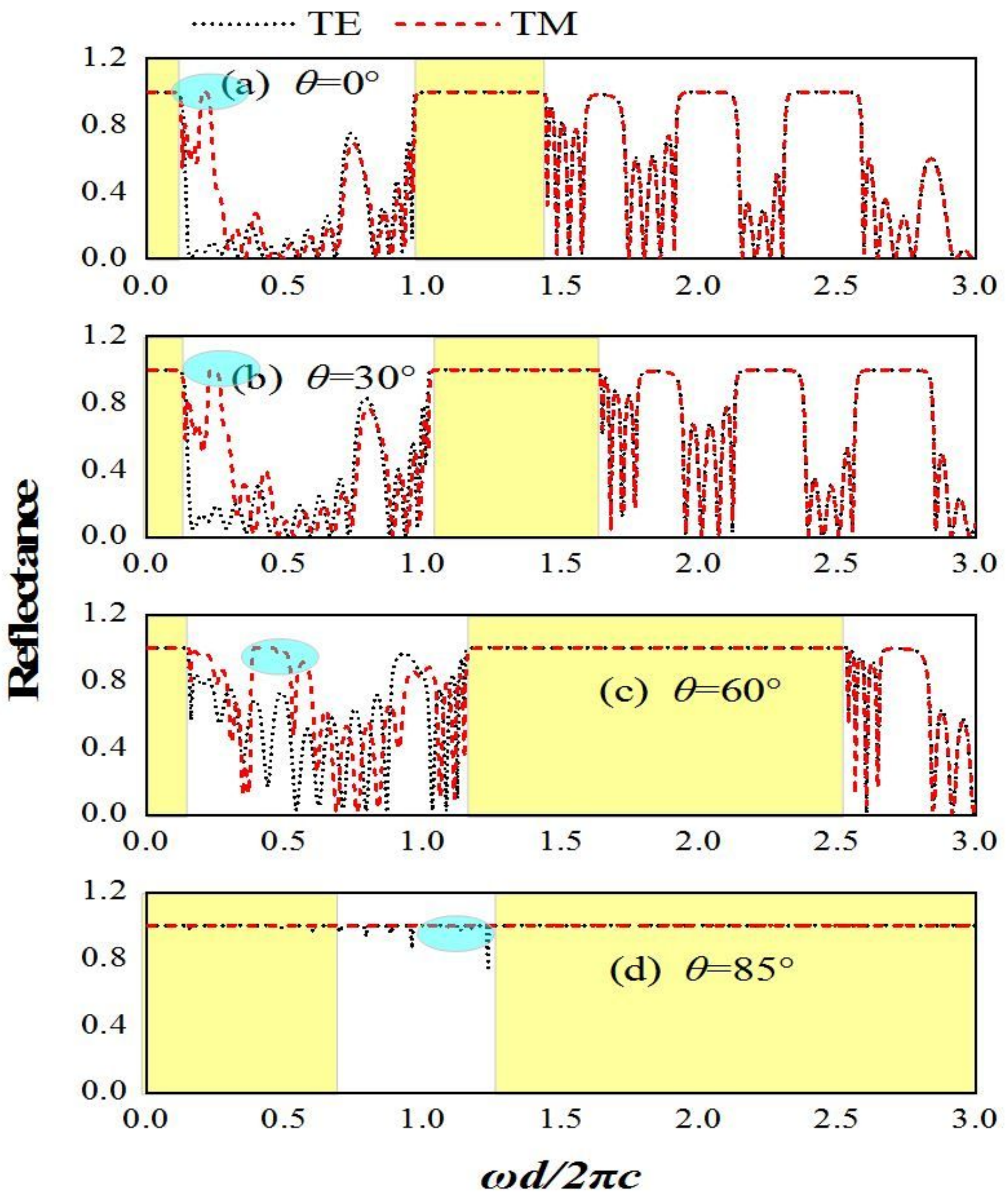

Figure 5

The reflection curves of the TM and TE waves for 1D PCPCs based on the IFT structure with different $\theta$ when $\mathrm{m}=2$. (a) $\theta=0^{\circ}$, (b) $\theta=30^{\circ}$, (c) $\theta=60^{\circ}$, and (d) $\theta=85^{\circ}$. 


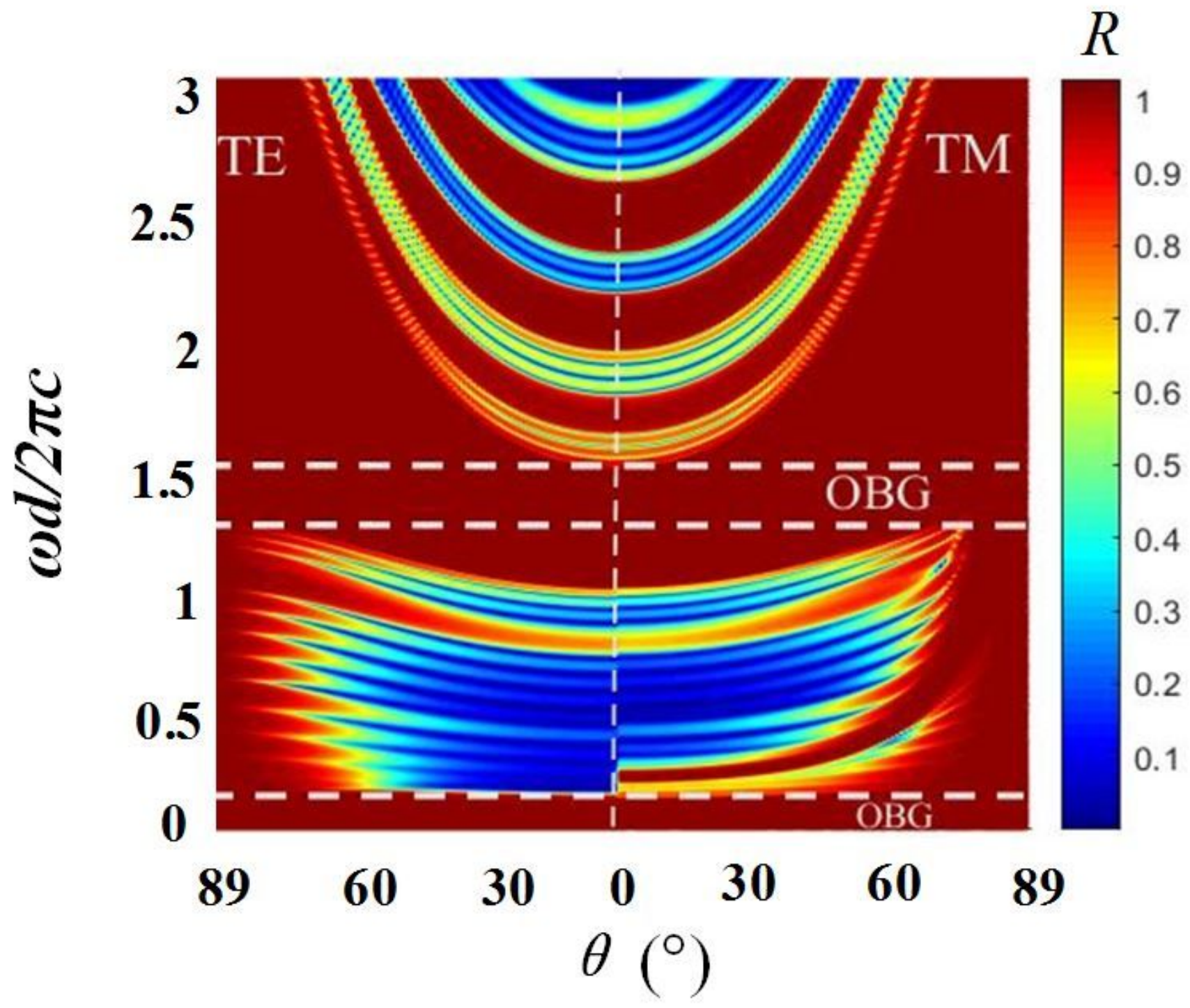

Figure 6

3D top view of $R$ diagram under different $\theta$, illustration of the relationship between $\theta$ and $R$ when $m=2$. 
TE - - . TM
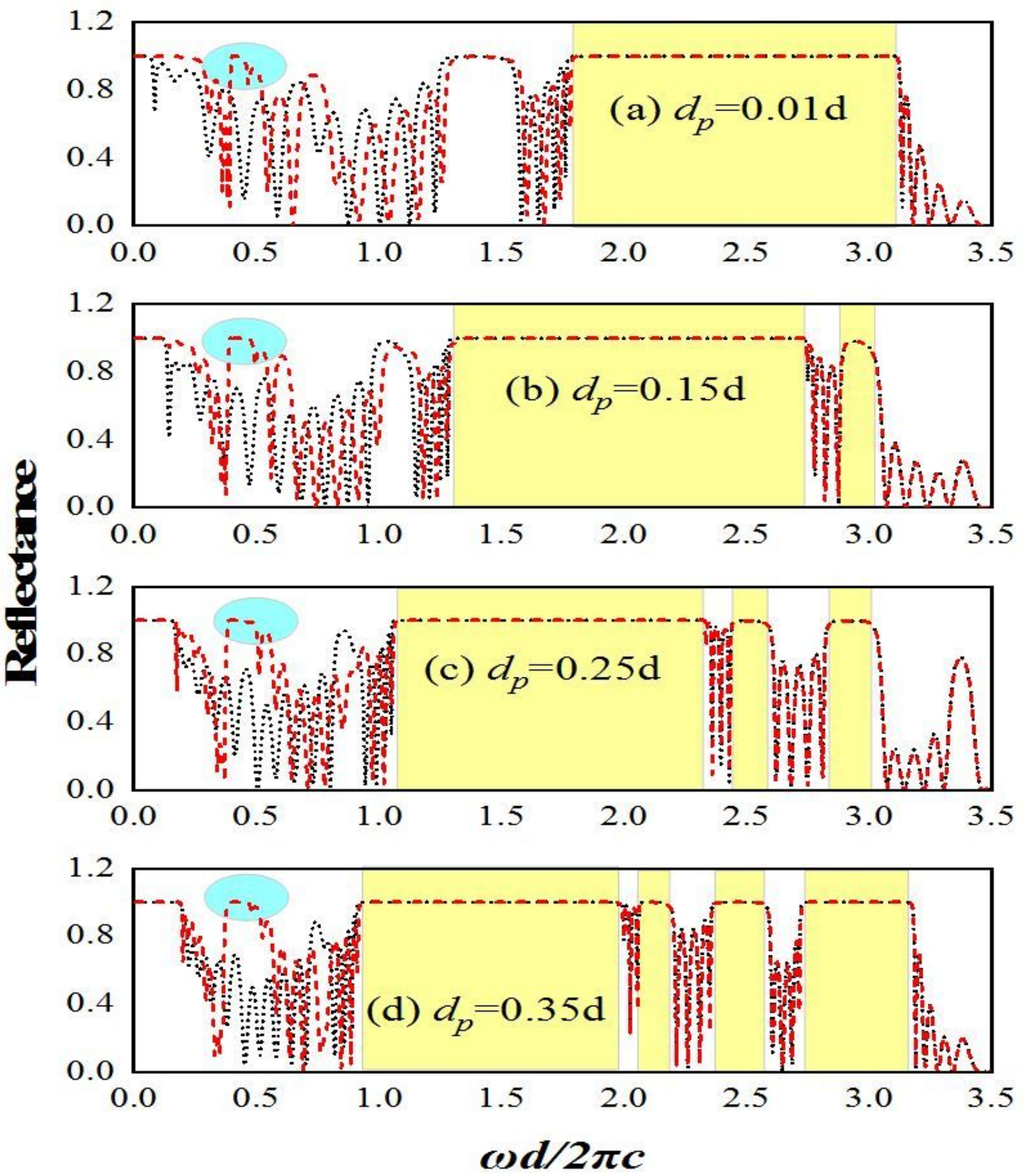

Figure 7

The reflection spectra of the TM and TE waves for 1D PCPCs based on the IFT structure with different dp when $\theta=60^{\circ}$ and $m=2$. (a) $d p=0.01 d$, (b) $d p=0.15 d$, (c) $d p=0.25 d$, and (d) $d p=0.35 d$. 


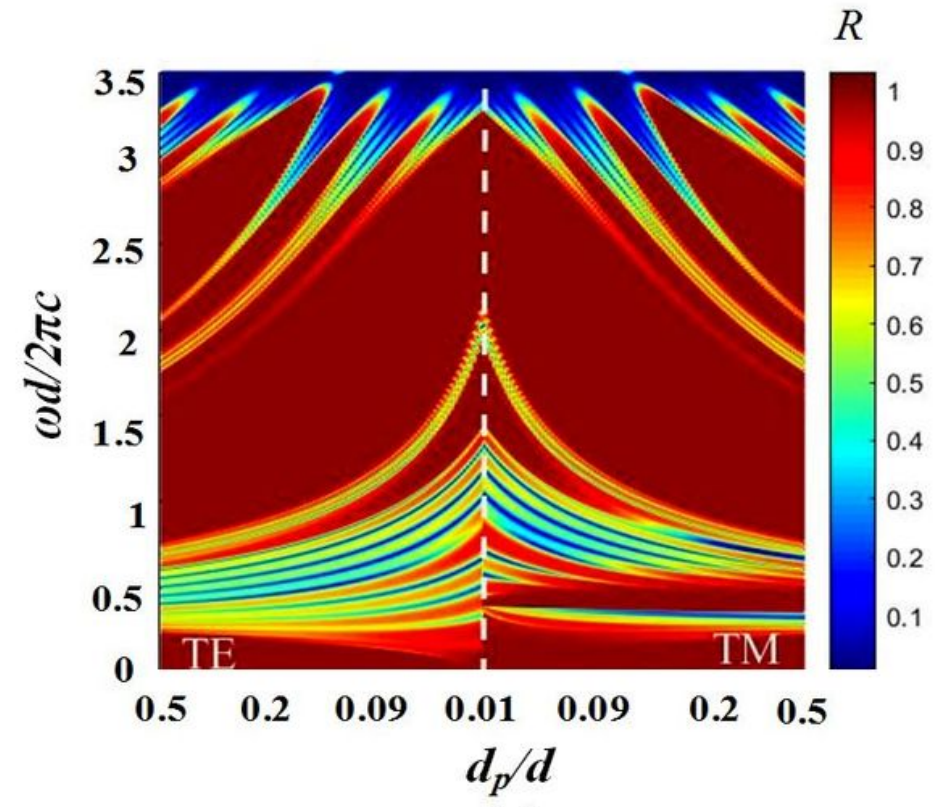

(a)

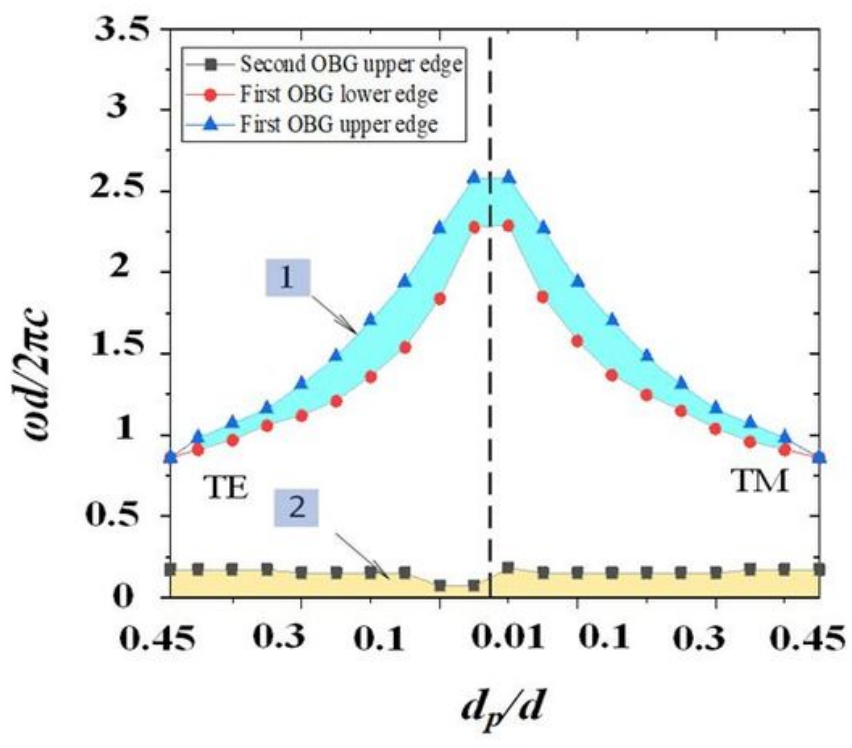

(b)

Figure 8

Diagram of the relationship between $d p$ and $R$ when $\theta=60^{\circ}$ and $m=2$, (a) 3D top view of $R$ diagram under different $\mathrm{dp} / \mathrm{d}$, and (b) diagram of $\mathrm{OBG}$ at different $\mathrm{dp} / \mathrm{d}$. 
TE - - . TM
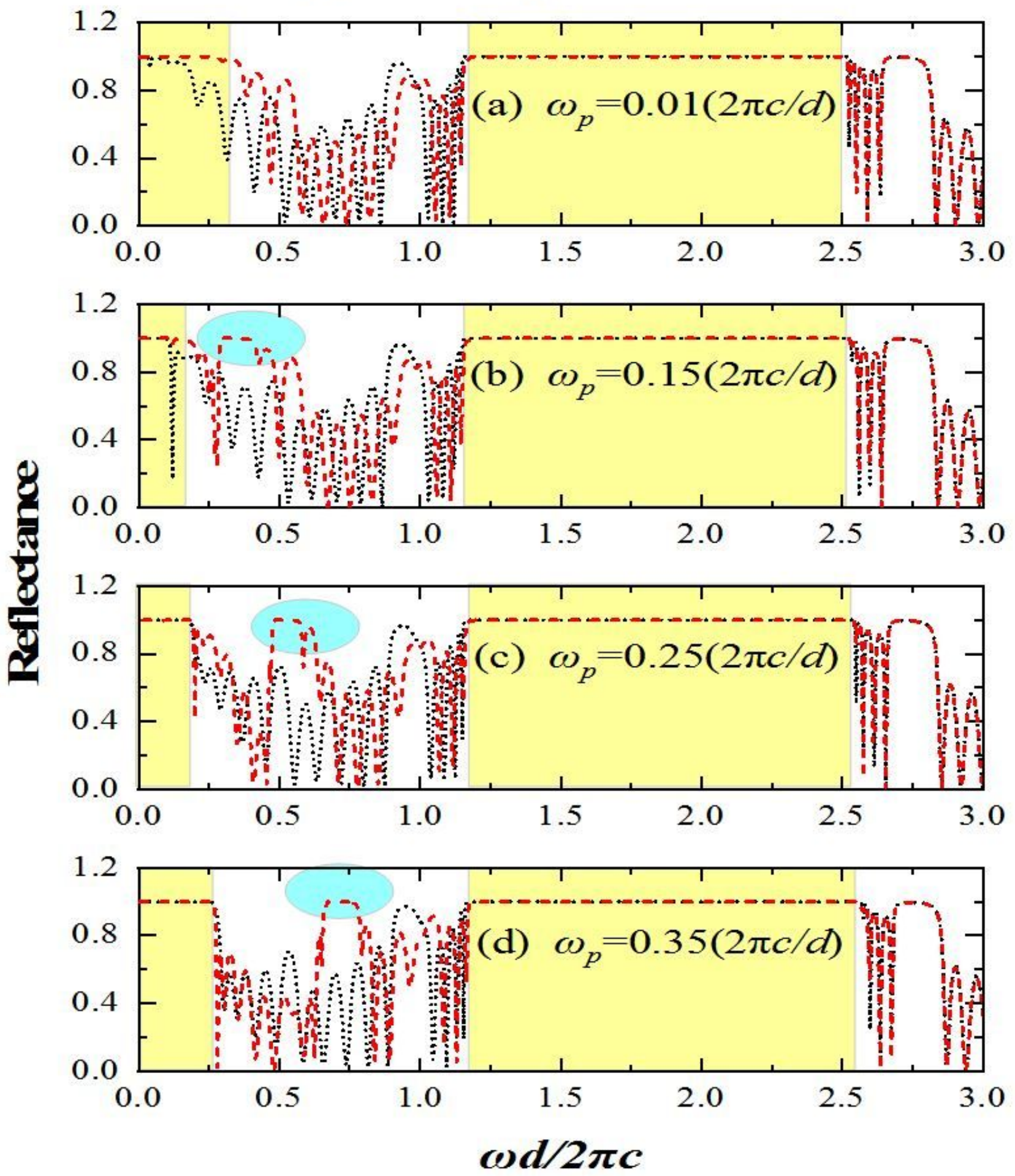

Figure 9

The reflection curves of the TM and TE waves for 1D PCPCs based on IFT structure with different $\omega p$ when $\theta=60^{\circ}$ and $m=2$. (a) $\omega p=0.01(2 \pi c / d)$, (b) $\omega p=0.15(2 \pi c / d)$, (c) $\omega p=0.25(2 \pi c / d)$, and (d) $\omega p=$ $0.35(2 \pi c / d)$. 


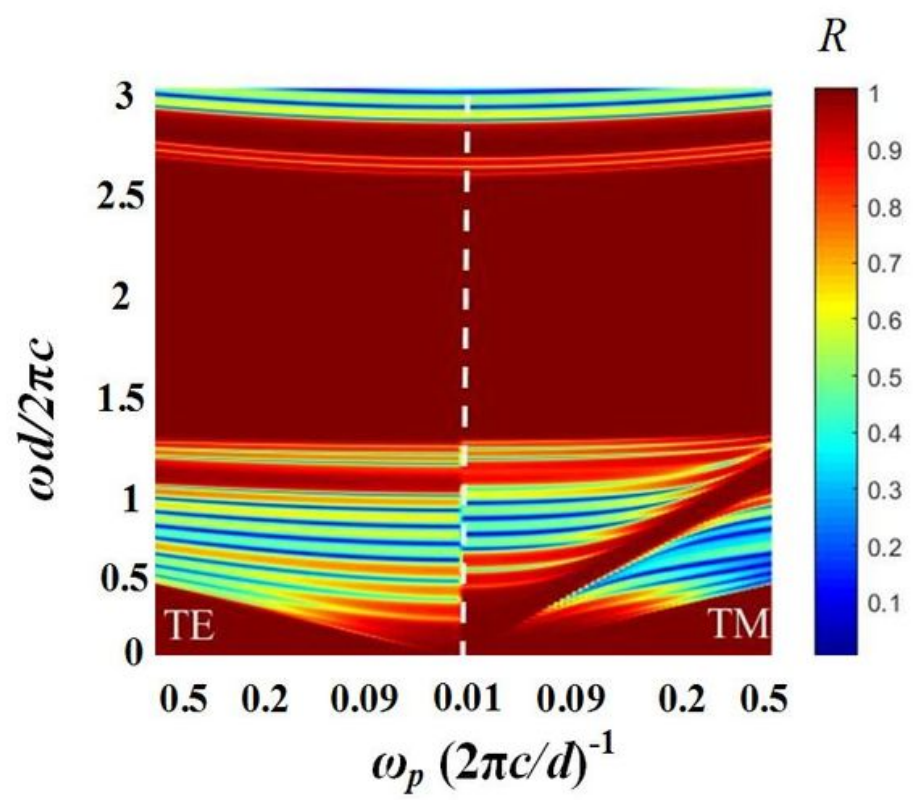

(a)

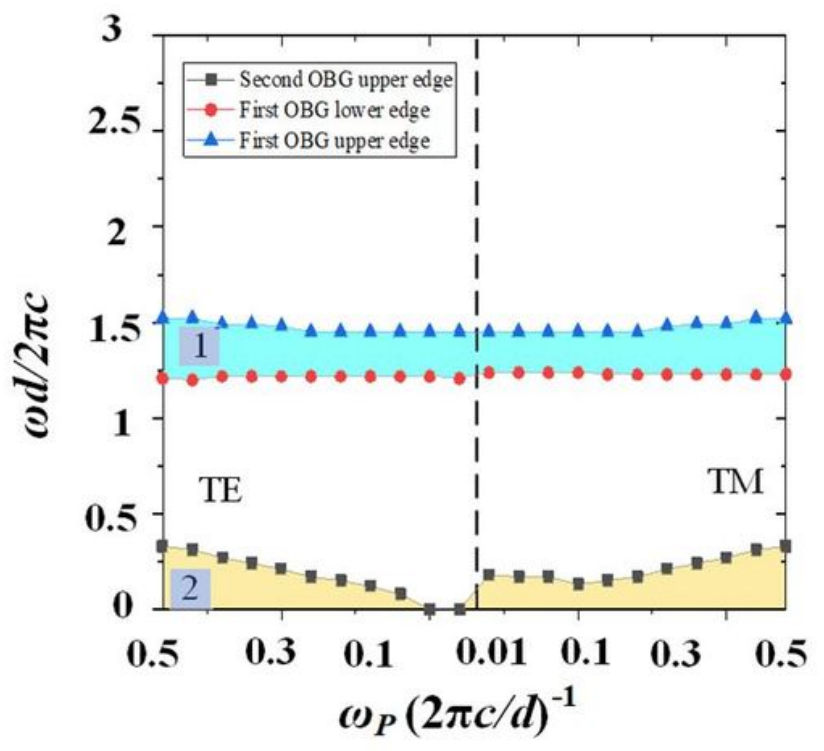

(b)

\section{Figure 10}

Confirm the truth of the relationship between $\omega p$ and $R$ when $\theta=60^{\circ}$ and $m=2$. (a) $3 D$ top view of $R$ diagram under different $\omega p$, (b) the diagram of $O B G$ at different $\omega p$ 